\title{
Mythe, métaphore, métamorphose et marches : l'igname chez les Lau de Malaita, îles Salomon
}

\section{Pierre Maranda}

\section{OpenEdition}

1 Journals

\section{Édition électronique}

URL : http://journals.openedition.org/jso/1418

DOI : $10.4000 /$ jso. 1418

ISSN : $1760-7256$

\section{Éditeur}

Société des océanistes

\section{Édition imprimée}

Date de publication : 1 décembre 2002

Pagination : 91-114

ISSN : 0300-953x

\section{Référence électronique}

Pierre Maranda, « Mythe, métaphore, métamorphose et marches : l'igname chez les Lau de Malaita, îles Salomon », Journal de la Société des Océanistes [En ligne], 114-115 | Année 2002, mis en ligne le 26 mai 2008, consulté le 22 mars 2021. URL : http://journals.openedition.org/jso/1418 ; DOI : https:// doi.org/10.4000/jso.1418

\section{(c) (i) $\ominus$}

Journal de la société des océanistes est mis à disposition selon les termes de la Licence Creative Commons Attribution - Pas d'Utilisation Commerciale - Pas de Modification 4.0 International. 


\section{Mythe, métaphore, métamorphose et marches : l'igname chez les Lau de Malaita, îles Salomon *}

par

Pierre MARANDA **

\section{RÉSUMÉ}

Comme l'indique Barrau, une synergie terre-mère s'effectue en Mélanésie par une relation d'équivalence entre deux récoltes, celles de poissons et celles de légumes. Les Lau de Malaita fondent cette convertibilité au moyen du mythe d'origine de l'igname. Dérobé aux esprits, le tubercule se transforme en poisson. Or ses écailles ressemblent aux coupures en lesquelles on détaille l'igname pour la planter. Jetées sur un tas d'ordures, les écailles germent et implantent ainsi l'igname à Malaita. On développe donc une métaphore en métamorphose qui génère un mythe d'origine, qu'on peut interpréter comme la charte des marchés traditionnels. Là, opératrices de la synergie terre-mer, les femmes transforment les produits marins en produits de la terre et inversement.

Mots-CLÉS : îles Salomon, Malaita, Lau, igname, mythe, métaphore, métamorphose, métaphore métamorphisante, marchés, synergie terre-mer.

\section{Introduction}

La reproduction d'un panneau de bois gravé sert de frontispice au texte de Jacques Barrau,

\begin{abstract}
Barrau has concisely made the point that both sea and land are harvested equivalently and yield interchangeable crops. The Lau of Malaita had long shared the same view and have expressed it in their myth of origin of the yam. Culture heroes steal tubers from the island of spirits that, angry, sink their get-away boat. Fallen in the sea, the yam turns into a big fish. Back home, the heroes catch the fish, throw its scales on a garbage heap. The scales, similar to the cuttings into which yam tubers are sliced for planting, germinate and thus bring yam to Malaita. The Lau have developed that metaphor (scales = cuttings) into a metamorphosis that generates a myth of origin. The myth can be interpreted as the charter of the institutionalized markets where, in everyday life, women implement the metamorphosis of fish into vegetables and vice-versa, and thus act as the operators of the land-sea synergy.
\end{abstract}

KEY-WORDS : Solomon Islands, Malaita, Lau, yam, myth, metaphor, metamorphosis, metamorphic metaphor, markets, land-sea synergy.

«Les hommes, les plantes et la mer en Océanie tropicale» (Barrau, 1967). On y voit l'« humide » et le « sec » (Barrrau, 1965) : à gauche, une pirogue en mer, occupée par trois hommes et

* Une version préliminaire de ce texte a fait l'objet d'une présentation dans le cadre du séminaire « Anthropologie et histoire de l'Océanie » (EHESS, Marseille) le 5 décembre 1995. Je remercie le Milton Fund de l'université d'Harvard, le Peabody Museum de la même institution, le National Institute of Mental Health (Washington, États-Unis) et le Conseil de Recherches en Sciences Humaines du Canada, organismes grâce auxquels j'ai pu faire mes terrains à Malaita.

** Département d'anthropologie, Université Laval, Québec, G1K 7 P4 $\quad$ (Fax +1 $418 \quad 656 \quad 28 \quad 31$; Pierre.Maranda@ant.ulaval.ca/pmaranda@videotron.ca). 
navigant au-dessus de poissons; au centre, un arbre à pain au tronc et aux branches évidées formant canal par où les poissons nagent jusqu'à une ouverture à la sortie de laquelle une femme, bien campée sur terre, " cueille » cette moisson marine. L'arbre à pain bissecte la gravure et fonctionne comme un médiateur entre terre et mer ( $c f$. ci-dessous, I-1-63). Barrau (1967 : 59) commente ainsi cette œuvre appartenant à sa collection : "Arbre à pain légendaire des îles Palau, dont le tronc et les branches creusées communiquaient avec la mer et permettaient aux insulaires de 'cueillir' les poissons $\gg$.

Le texte qui suit résonne en écho complémentaire aux écrits de Barrau, en particulier ceux sur l'igname. J'y montre comment, pour les Lau (prononcer " lao ») de Malaita, îles Salomon, un «sec» - l'igname — se métamorphose en « humide » - un poisson - pour se re-métamorphoser en igname. Cette métamorphose se voit répétée quotidiennement sur les marchés traditionnels où se rencontrent en synergie gens de la terre (les jardiniers montagnards de Malaita) et gens de la mer (les pêcheurs vivant sur les îles artificielles de la Lagune Lau). Là, le médiateur n'est pas l'arbre à pain, mais la femme nubile qui, dans ces marchés côtiers, transforme indéfiniment en les échangeant les uns contre les autres, et depuis des générations, des légumes en poissons et des poissons en légumes.

Voyons d'abord en première section un échantillon des mythes d'origine de l'igname à Malaita. Suivra un sommaire de données ethnographiques pertinentes. En troisième lieu, une analyse succincte mènera à la quatrième section qui fournit une interprétation de ce mythe fondateur de la synergie entre le « sec » et l'«humide $»$.

\section{Présentation d'un échantillon des mythes d'ori- gine de l'igname}

J'ai recueilli au cours des vingt-trois dernières années (1966-1989) et de divers séjours sur le terrain dix-huit variantes du mythe d'origine de l'igname à Malaita, sans compter les reprises, à quelques années d'intervalles, par les mêmes narrateurs, et sans compter celles recueillies par mon collaborateur, Luc Lafrenière. Je précise que par variante, j'entends la leçon particulière d'un conteur dans le contexte de la cueillette. J'appelle version la répétition d'une variante par un conteur sans qu'il n'en modifie substantiellement ni la structure ni le contenu. J'appelle texte l'ensemble des variantes sur un sujet donné, tel l'origine de l'igname.

Ici, la présentation des données, restreintes à trois variantes dont les deux premières sont résumées, va de la plus simple et rudimentaire à la plus complète de ce petit échantillon. La numérotation, sans portée analytique, renvoie à celle adoptée dans une monographie qui portera sur les dix-huit variantes. Les notes en retrait comportent des commentaires exégétiques ou explicatifs, les compléments ethnographiques et l'analyse elle-même venant ensuite. On se reportera à la carte 1 pour situer les lieux de l'action et à la carte 2 pour situer les narrateurs des trois variantes.

\section{Première variante, en provenance de l'intérieur (kwara'ae)}

On ne peut cultiver l'igname au-delà d'une certaine altitude (Bonnemaison, 1997 : 198 ss). À Malaita, on ne la trouve donc que sur les versants côtiers. Au nord, les " gens de la terre ", habitants de la grande île face à la lagune lau et en interaction avec ceux qui y vivent sur les îles artificielles qu'ils y ont construites depuis plus de six cents ans (Maranda et Köngäs Maranda, 1970 ; Maranda 1979, 1982a, 1985 et 2001 ; Granada Televison, 1987), ne prisent pas ce tubercule autant que les " gens de la mer ». Cela explique probablement, en partie du moins, que, en dépit de nombreuses tentatives parmi les «gens de la terre », je n'aie réussi à recueillir parmi eux que la variante qui suit du mythe d'origine de l'igname ${ }^{1}$.

On a là une variante « réaliste » et très courte, racontée par un homme de l'intérieur, un Kwara'ae (les Kwara'ae vivent dans la grande île, à l'extrême limite méridionale de la lagune lau). Je l'ai prise à la dictée du narrateur, en mai 1976, à bord d'un caboteur assurant la liaison Honiara (Guadalcanal) - Auki (Malaita) en dialecte baelelea, dialecte proche du lau et que le conteur maîtrisait fort bien, en présence d'autres hommes comprenant aussi le baelelea. Ce narrateur, homme dans la quarantaine, vigoureux et conscient des traditions de son ethnie, passa le reste du trajet à nous expliquer, aux autres passagers et à moi, comment les Malaitains, qui avaient toujours bien vécu laissés à eux-mêmes, pouvaient fort bien se passer de tous ces objets techniques qu'apportent les blancs. Comme pour les autres variantes, la numérotation en segments n'a aucune prétention analytique; elle ne vise que la commodité à fins de renvois. 


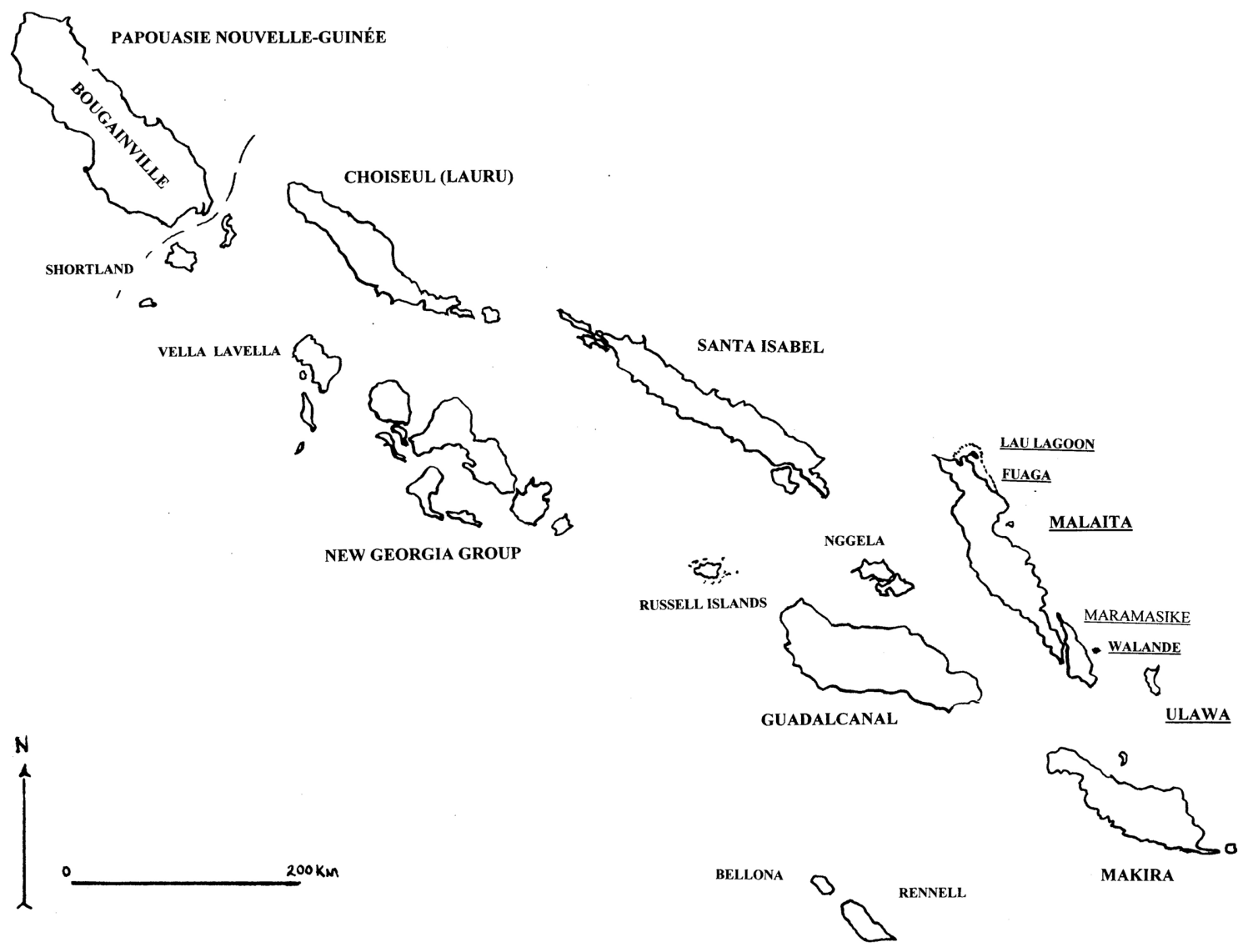

CARTE 1 : carte sommaire de l'archipel des îles Salomon. Les noms soulignés indiquent les déplacements de l'igname.

I-10-1 L'igname a dérivé au gré des courants vers Malaita.

Effectivement, l'igname flotte si on la met à l'eau.

I-10-2 Un jour, elle s'échoua sur le rivage.

I-10-3 On l'y a aperçue, l'a ramassée, l'a goûtée.

I-10-4 La trouvant bonne à manger, on l'a plantée et on la cultive depuis.

I-10-5 Voilà comment a commencé l'igname à Malaita.

Deuxième variante, en provenance de Maana'oba (île naturelle à l'intérieur de la lagune lau)

Variante enregistrée à Tauba-sur-mer le 24 juillet 1976, racontée par Faka'iia, aristocrate de Maona'oba, en présence de Lilikai (conteur d'une autre variante). Homme dans la soixantaine, consciencieux, sobre en paroles et en gestes, il dit tenir sa leçon d'un homme de Gounaasi (littéralement, " la tête de la mer », c'est-à-dire « outremer ») où l'igname apparut pour la première fois, au temps originel. Les quelques hommes présents dans l'aire des hommes se regroupèrent pour entendre le conteur mais ni ne lui posèrent de questions ni ne firent de commentaires. Mon résumé suit le texte de près.

\section{I-7-1 À Gounaasi,}

En réponse à une question après la narration, le conteur dit que Gounaasi s'appelle maintenant Walande ; il s'agit d'une petite île sur la côte est de Petite Malaita (Maramasike, carte 1).

un homme pêche à la ligne en haute mer.

Par opposition à la pêche côtière ou lagunaire, celle que pratiquent les autres héros de ce mythe.

I-7-2 Il donne de la ligne, beaucoup, beaucoup

de ligne. C'était l'heure où l'igname mangeait.

L'heure où le poisson mange : expression courante chez les Lau pour désigner le moment propice pour une bonne pêche. Le narrateur stipule donc l'équation igname $=$ poisson . 


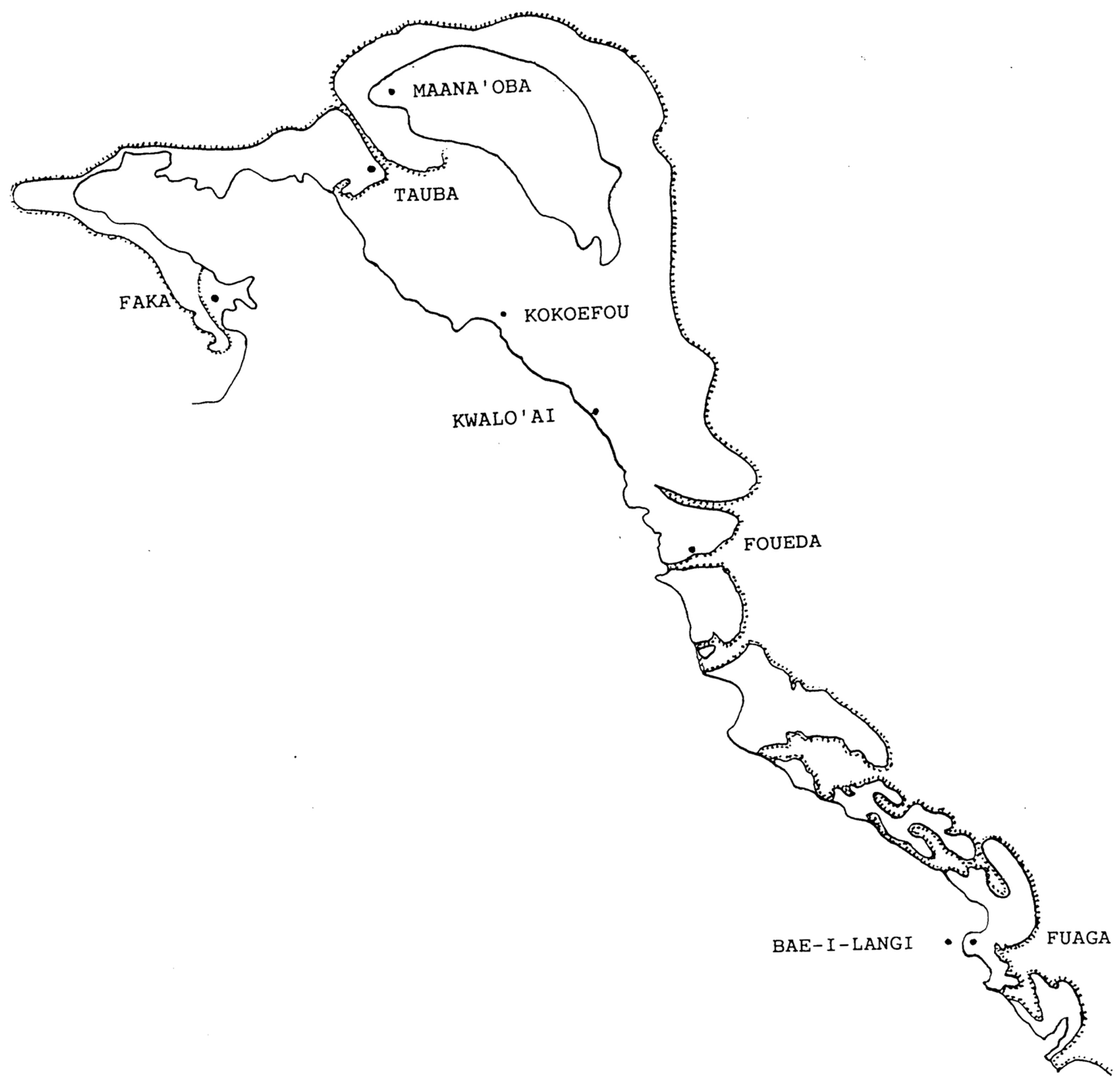

KWARA ' AE

Carte 2 : carte sommaire des villages où vivent les narrateurs des variantes. Pour les différentes tribulations des ravisseurs de l'igname depuis le sud-est, voir Carte 1.

I-7-3 L'igname mord à l'hameçon, sous forme d'alia. Le nom de l'igname est alia, c'est-à-dire un poisson [insiste le narrateur].

$C f$. infra, I-1-15. On verra plus loin la relation d'association par similarité entre écailles d'alia et découpures d'ignames pour la plantation.

I-7-4 Le pêcheur ramène sa ligne, tire, tire, tire, tire longtemps. Il finit par hisser le poisson à bord de son embarcation.

I-7-5 Le pêcheur rentre au village, écaille sa prise

I-7-6 Une fois tout l'alia dépouillé de ses écailles, une de celles-ci dit au pêcheur: «plantemoi ».

L'écaille, déjà, douée de parole, et non pas l'igname comme dans d'autres variantes, donne des instructions à l'homme.

I-7-7 Il la plante, et elle se transforme en igname.

I-7-8 Le pêcheur pose un tuteur, comme l'écaille le lui avait demandé. Il rechausse la plante, il la promulgue tabou, suivant, en toutes ces opérations, les instructions données par l'écaille. Une fois la plantation bien fournie, il déterre les ignames. Et c'est ainsi que l'igname a commencé. 
Je dois citer ici une variante recueillie par de Coppet (1976 : 170-171), dont on verra immédiatement la pertinence.

Le mythe d'origine de l'igname raconte :

« Deux frères qui s'appelaient Epie la bonite, après avoir joué de malchance plusieurs jours de suite à la pêche, voient flotter sur la mer [cf. I-10, supra] une chose étrange. Ils s'en approchent en pirogue, mais la chose plonge soudain. Ils attendent qu'elle remonte et, sitôt qu'elle fait surface, l'accrochent. Elle replonge, entraînant la liane qui la relie au bambou. Après une longue bataille [cf. I-7-4], les deux frères réussissent à la tirer dans leur pirogue et mettent le cap sur le rivage. A terre, ils en coupent une tranche, l'intérieur en est d'un beau blanc transparent. Ils essayent de la planter, elle pousse et se multiplie. C'est la première igname ».

Et de Coppet continue : Ainsi fut capturé en mer, comme une bonite [cf. I-1-14, infra], ce tubercule qui donne chaque année la nourriture de base des gens de la côte. Il mûrit dans la terre et sa tige grimpante s'enroule autour d'une rame de bambou; de même la bonite est pêchée à la ligne accrochée à une canne de bambou. On comprend mieux pourquoi le jeu des flûtes de Pan est interdit pendant les rituels au jardin. En effet, souffler dans les bambous de la flûte, c'est tendre depuis le bambou une ligne mélodique pour séduire les filles (poissons), c'est-à-dire préjuger de la capture des ignames (poissons) au bout des rames de bambou ${ }^{2}$.

Troisième variante, en provenance de Foueda (passe Makwanu, au centre de la lagune, voir carte 2)

Variante recueillie à la machine à écrire le 11 mars 1967 dans la paillote de l'ethnographe, à Foueda (le second des îlots artificiels construits dans la lagune, à son centre géographique, ancestral, religieux et politique, dans la passe Makwanu). Racontée par Ramoagalo, chef du clan rere, le clan le plus ancien et le deuxième en importance démographique de la Lagune. Ramoagalo, alors dans une jeune et exceptionnellement vigoureuse soixantaine, éminait de par toute Malaita septentrionale par ses grandes connaissances, reconnues comme incontestables. Les nombreuses alliances matrimoniales de son clan avec d'autres du nord de la grande île lui faisaient des « fils » ou " neveux » qui venaient périodiquement lui rendre des visites de courtoisie ou avoir recours à ses bons offices pour des affaires délicates ou des interprétations des traditions locales. J'ai pu aussi constater à plusieurs reprises comment d'autres clans se reportaient également à lui pour trancher des litiges requerrant sagesse et savoir, et pour ses offices de médiateur en cas de conflits entre ceux qui avaient pris les armes les uns contre les autres mais s'adressaient à lui avant de passer à l'attaque.

La prédiction de la présence, un jour, de blancs dans la lagune « qui voudraient tout savoir de la façon de vivre des Lau », faite par son oncle maternel quelque quarante ans avant notre arrivée, avait incité Ramoagalo à faire des stages d'étude parmi tous les «savants» des divers clans de la grande île. Il avait alors environ vingt ans. Depuis, il se préparait à recevoir la visite de ceux que lui avait annoncés son oncle.

Son frère Daui - dont Ramoagalo avait supplanté l'aînesse par dynamique personnelle -, savant mythologue lui aussi, guérisseur renommé et prêtre de lignée (ai), assistait à la dictée, de même que le fils de Ramoagalo, Aluta, headman de la lagune et lui-même homme d'une très grande intelligence et d'un savoir traditionnel remarquable. La variante qu'il raconta, Ramoagalo l'avait entendue lors d'un voyage au sud, il y avait plus de trois décennies, a-t-il dit.

Alors que j'ai résumé plus haut deux des dixhuit variantes du mythe de l'igname, la traduction de la suivante, quasiment littérale, colle à la leçon. Le découpage en phrases correspond aux pauses du narrateur qui a su s'adapter rapidement à la cadence de frappe. Quant à la numérotation en segments, elle n'a aucune prétention analytique, ne visant que la commodité à fins de renvois.

\section{I-1}

I-1-1 Des hommes de trois villages d'une petite île située dans le détroit qui sépare les deux Malaita et appartenant à Petite Malaita, partent en expédition dans une grande barque vers Ulawa.

On apprendra plus bas le nom de la petite île: Alai, à l'est de Maramasike. Ulawa : île plus au sud ; voir carte 1 et la prière en provenance d'Ulawa dans laquelle on assimile l'igname au poisson (citée infra (7).

I-1-2 Suit la nomenclature des endroits qu'ils doublent en mer.

Je reviendrai plus loin sur le sens de ces énumérations, fort fréquentes et significatives dans les mythes malaitains.

I-1-3 Après un jour et demi, ils abordent à Ulawa, où ils sont bien reçus. On leur apporte de la nourriture dont de l'igname.

2. Faute d'espace, je ne développerai pas ici cette relation d'équivalence métaphorique filles/poissons/ignames et ligne/tige de l'igname, dans le contexte de la représentation du monde chez les Lau. 
Dans les dialectes de l'intérieur de Malaita septentrionale, un même terme désigne " nourriture » et « igname », et cela bien qu'on n'y cultive que fort peu l'igname. D'ailleurs, en pidjin, kai ou kaikai signifie " nourriture, manger ». Kai asi ainsi que kairogi désignent des espèces de patate, kai'ai, le manioc.

I-1-4 Les visiteurs se régalent d'ignames, dont ils apprécient fort le goût.

I-1-5 Après quatre jours chez leurs hôtes, ils disent:

- Nous rentrerons chez nous dans un jour ou deux.

Ce diseur de mythes précise toujours les laps de temps dans l'action. Et, comme la plupart des conteurs lau attitrés (et de nombreux Mélanésiens, e.g., les Maenga [M. Panoff 1970a]), il ne manque pas de chiffrer chaque fois que l'occasion se présente: nombre de guerriers dans une armée, nombre d'embarcations et de marins dans une flotte, nombre de brasses de monnaie de coquillage et de dents de dauphins dans un finisi « prix du sang », etc.

I-1-6 Deux des visiteurs, dont les noms sont

Faneakwaakwa l'aîné et Faneakwaakwa le cadet, disent :

Faneakwaakwa se décompose en deux termes dont le premier, fane, de l'accord quasi unanime des Lau intéressés par l'étymologie, signifie "rapide au tir à l'arc" et le deuxième, aakwaakwa, selon l'acception la plus commune, désigne un poisson minuscule ( $c f$. mud-hopper) qui se déplace à la pointe des marées. On prendra toutefois note infra de débats sur cette ethnoétymologie.

- Ce soir, si la lune est claire, allons voler des ignames, cette chose que nos hôtes nous ont interdit d'emporter chez nous.

Interrogé après son récit, sur la raison de cet interdit, le narrateur répondit qu'on était d'accord pour que les visiteurs rapportassent avec eux des ignames cuites mais non des crues, ce qui évidemment ne pouvait faire leur affaire vu qu'on ne peut planter de tubercules cuits.

\section{I-1-7 Ils exécutent leur plan, cachent les ignames} dans la barque, et partent en pleine nuit.

I-1-8 Ils sont rendus au large lorsque l'esprit d'Ulawa se rend compte du vol :

I-1-9 il leur dépêche un ouragan qui les fait chavirer avec les ignames.

I-1-10 Les hommes remettent la barque à flot et le calme revient sur la mer mais ils ne récupèrent pas les tubercules.
I-1-11 Doublant de nouveau des lieux repérés, les héros rentrent chez eux bredouilles. Les deux Faneakwaakwa disent :

- Quel dommage! Cette chose que nous avions apportée, elle a sombré. Nous ne l'apportons pas.

I-1-12 Un mois se passe, puis deux, puis trois.

I-1-13 Les deux Faneakwaakwa se disent l'un à l'autre :

- Allons, prenons un filet.

Filet de type abeteu, qu'on pose à plat sur le fond de la lagune et qu'on surveille du haut d'un arbre ou d'un échafaudage pyramidal de perches construit à cette fin (airia).

et pêchons à la pointe Kwaiakwa.

Kwai « ramener le filet »; akwa « espèce de poisson »; cf. akwaakwa, deuxième partie du nom des héros.

I-1-14 Ils attendent de la bonite, voient passer un roomaa.

Dans cette variante-ci, les héros vont tout simplement à la pêche sans autre intention, alors que dans quelques autres ils vont pêcher explicitement pour récupérer l'igname, selon les instructions de l'esprit du clan.

Roomaa « poisson plat à deux grands yeux »; cependant, le narrateur dira plus loin (I-1-27) qu'il s'agit d'un unugwaila et, quelques années plus tard lorsque je révisai son récit avec lui, il insistera qu'il ne s'agissait pas d'un roomaa mais bien d'un alia (Cheilinus undulatus, Humpheaded Maori Wrasse), comme dans toutes les autres variantes où l'igname est un poisson.

I-1-15 C'était le poisson dans lequel l'igname s'était métamorphosée lors du naufrage.

Le vocable lau pour "se métamorphoser en » est hau 'ana.

C'était un poisson immense, mesurant cinq brasses.

I-1-16 Ce poisson souffle sur leurs yeux, les deux hommes tombent de sommeil, et le poisson continue son chemin.

I-1-17 À leur réveil, ils constatent :

- Ce poisson-là s'est sauvé.

Ils rentrent au village avec le filet.

I-1-18 Deux jours passent ; le troisième, les deux hommes disent :

- Allons-y, postons-nous de nouveau pour attendre ce poisson-là.

I-1-19 Ils répètent la manœuvre. Ils aperçoivent le poisson qui s'approche. Ils disent :

- Cette chose-là, ce poisson-là, il vient ici, le voici tout proche. 
I-1-20 Le poisson à cinq brasses d'eux, les deux s'endorment. À leur réveil, ils se rendent compte que le poisson s'est dérobé, et ils rentrent au village avec le filet.

I-1-21 Ils laissent s'écouler trois jours, le quatrième, ils repartent, Faneakwaakwa aîné et Faneakwaakwa cadet.

C'est la première fois dans le récit que le narrateur mentionne la relation d'aînesse entre les deux Faneakwaakwa ; auparavant il se contentait de dire "les deux Faneakwaakwa ».

I-1-22 Une troisième fois, ils répètent la manœuvre. Vers 16 heures,

Abalili en lau correspond au début de la fin de l'après-midi.

ils aperçoivent le poisson qui vient vers eux. Ils disent :

- Cette chose-là revient de nouveau vers ici, et elle vient proche.

Le poisson, de nouveau à cinq brasses d'eux, les plonge encore dans le sommeil. À leur réveil, le poisson a disparu. Ils disent :

- La prochaine fois, que faire afin que nous restions éveillés et que nous puissions tuer cette chose-là qui vient vers nous ?

I-1-23 Ils ne savaient pas encore que le nom de cette chose n'était aucunement nom de poisson.

I-1-24 Là donc, ces deux hommes dormirent de nouveau, et le poisson s'échappa de nouveau. Ils dirent :

— Hé ! Le poisson s'est échappé !

I-1-25 Là donc, ils ramènent le filet au village. Ils attendent quatre jours. Le cinquième, ils reprennent leur filet, ils repartent. Une fois le filet en place, Faneakwaakwa l'aîné dit à son cadet, Faneakwaakwa cadet :

- Si tu prends garde, si cette chose-là arrive, nous parlerons - nous aurons beau nous appesantir, nous parlerons et cela nous gardera éveillés.

Ils conversent donc. Ils voient le poisson s'approcher, ce poisson qui les fait dormir; ils conversent et le poisson s'engage dans le filet.

I-1-26 Ils capturent le poisson. Ils l'examinent bien, ils disent : dormir.

- C'est, en effet, le poisson qui nous a fait

\section{I-1-27 Le nom de ce poisson est unugwaila.}

Sur le nom du poisson, voir I-1-14.

I-1-28 Ils apportent leur prise au village, entrent avec elle dans l'aire des hommes, en raclent les écailles.
Comportement normal: au retour de la pêche, les hommes rentrent toujours au village par l'aire des hommes - les femmes ne doivent pas apercevoir les agrès de pêche, utilisés exclusivement par les hommes, d'ailleurs seuls autorisés à pêcher.

I-1-29 Quelques écailles tombent à la mer, quelques-unes tombent sur un tas d'ordures - un tas d'ordures comme celui que tu vois là-bas.

Le conteur désigne un tas près de la paillote. Il s'agit de tas d'ordures qu'on ne jette pas à la mer, comme la plupart des déchets (autrefois tous « naturels », biodégradables), mais d'ordures qu'on laisse se dégrader sur l'îlot artificiel à cause de leur charge symbolique. On a vu que, dans I-7-5 à 8 , une écaille donne au pêcheur les instructions pour qu'il la plante et sur la façon de cultiver le tubercule.

I-1-30 Ils prennent donc ce poisson, le font cuire, le mangent.

I-1-31 Une écaille de ce poisson demeure sur le tas d'ordures, y demeure neuf jours.

I-1-32 Au dixième jour, cette écaille avait pourri et les deux hommes voient qu'une chose germe.

I-1-33 Et elle se transforme en igname. Cette chose pousse et elle est rouge, elle est blanche, elle est bleue.

Couleurs de la chair de différentes espèces d'ignames.

\section{I-1-34 Les deux hommes disent :}

- Cette chose-là, qui a chaviré avec nous, peut-être s'est-elle transformée en poisson ?

I-1-35 Cette igname continue ainsi pendant cinq mois. Au sixième mois, ses feuilles sont sèches, Dele, en lau, signifie « cuit, sec, mûr ».

et elle est mûre. Elle est mûre, bonne à manger. Et cette igname reste là, et fait des pousses à son tour.

Il s'agit, bien sûr, ici comme dans d'autres variantes où elle se reproduit entière, d'une igname particulière car, disent les Lau, ce tubercule ne se reproduit pas de lui-même et planté entier, il resterait petit et trop coriace pour offrir un aliment satisfaisant. Il faut le découper en morceaux qu'on plantera et le cultiver, lui mettre des tuteurs, etc., pour qu'il porte des fruits comestibles. Cependant, Barrau (1956 : 64) écrit que, en Nouvelle-Calédonie, en ce qui avait trait aux « ignames de compétition qui atteignaient jusqu'à $3 \mathrm{~m}$. de longueur, [...] on ne plante d'ailleurs jamais pour obtenir de telles ignames une section de tubercule mais toujours un tubercule entier » (cf. aussi Bonnemaison 1991: 79, 
1996 : 228, $244 ; 1997$ : 202 ss sur quelles ignames on plante entières et quelles, en sections, au Vanuatu).

I-1-36 Et cela dure trois mois. Cette nouvelle igname atteint également la maturité. Elle gagne tout l'espace disponible.

I-1-37 Cette chose-là est mûre, dit un homme du nom de Maioa.

Ethno-étymologie de ce nom: mai «vers ici »; oa « partager ».

I-1-38 Et il dit :

- Vous autres, laissez faire, moi, je vais prendre de cette chose.

Cette chose n'avait pas encore de nom. Cet homme Maioa prend donc une igname et la fait cuire. Maioa l'épluche bien, ensuite il la rompt et la mange.

I-1-39 Et là, il se trouve repu.

I-1-40 Les gens d'Alai disent :

- Si cette chose était mauvaise, cet homme, qui aurait mangé une chose mauvaise, il serait mort.

Il en mange à nouveau au matin, et puis vient la nuit. On dort; à l'aube, ces gens-là parlent ainsi :

- Si nous prenions vingt ignames et si nous en mangions?

Ils font donc cuire vingt ignames, ces gens-là, ils mangent de cette igname et ils sont tous repus.

I-1-41 Les hommes en étaient heureux à se rouler par terre, tellement ils se sentaient repus. Et ils disaient :

- Il ne faut pas que, à l'avenir, les femmes mangent de l'igname tant que l'esprit d'Alai ne nous aura pas instruits là-dessus.

I-1-42 Le nom [de cette chose] est igname, on a prononcé que cette chose serait appelée du nom d'igname.

I-1-43 Et si nous en prenons, nous devons d'abord en donner à l'esprit pour qu'il en mange en premier. Ensuite, nous donnons la deuxième igname au prêtre pour qu'il en mange. Ensuite, nous autres, nous pourrons en manger, seulement après l'esprit et le prêtre. Alors homme et femme pourront manger de l'igname.

I-1-44 Il ne faut pas qu'on laisse tomber l'igname par terre; si on la laisse tomber il faut tout de suite s'écrier: "Cette igname est tombée dans la main de Maioa », autrement, cette igname affligera cette personne d'enflures.

I-1-45 Et il en est encore ainsi aujourd'hui. Aux premiers temps, les gens d'Alai, ils interdi- saient aux femmes en règles de manger de l'igname.

On verra plus loin d'autres tabous quant au comportement envers l'igname.

I-1-46 Là donc, la nouvelle de l'igname se répand à Abuala et elle se répand à Maanafou,

Villages dans le voisinage d'Alai, Maramasike.

et on entend parler de cette chose vraiment bonne, qui s'appelle igname.

I-1-47 Tout le monde dans l'intérieur de Petite

Malaita et tout le monde de la région d'Abunabasi est mis au courant, et tout le monde de Mamalau aussi. «Cette chose est suprême, ce poisson que les deux Faneakwaakwa ont pris et qui porte nom unugwaila, et dont ensuite ils ont jeté les écailles sur un tas d'ordures pour les voir se transformer en igname ». Et ils disent :

\section{I-1-48 Aménageons deux grands falisi}

Nom de la plantation consacrée à la culture de l'igname, à l'exclusion de toute autre plante. On appelle la saison de l'igname uu ni falisi (uu " unité de temps, saison, période... ».) ; ni marque de génitif.

I-1-49 « dans ces deux terrains appelés les deux Alaitolo et plantons-y l'igname ».

Il peut s'agir ici de Alai-en-montagne ou de Ala-en-montagne, c'est-à-dire sur l'île de Maramasike.

I-1-50 Ils en font donc ainsi. Et ils démarquent ces deux terrains, et ils les défrichent, et ils préparent le sol, et ils les fouissent au bâton, et ils plantent dans ces deux plantations 20000 découpures d'igname.

Les rendements des ignames originelles varient d'un conteur à l'autre.

La plantation commence à pousser et on y met les tuteurs, et ça pousse, et on attache les plants d'igname aux tuteurs. Les deux plantations sont bien fournies.

I-1-51 Après trois mois, l'igname est mûre. On annonce alors aux habitants d'un village voisin, Abuala, qu'ils pourront se procurer dix ignames à raison de dix brasses de monnaie de coquillages et de mille dents de dauphin.

Ce prix est excessif par rapport au marché contemporain de l'igname : traditionnellement, et jusqu'aujourd'hui, on obtient mille ignames pour une brasse de monnaie de coquillage. Le prix demandé dans ce récit correspond à la prestation monétaire moyenne pour l'obtention d'une épouse - ainsi, un lot de dix ignames aurait la même « valeur » qu'une femme. Quant 
à des données sur le système monétaire de Malaita septentrionale, $c f$. Maranda, 1982: 31-37 ; Maranda in Godelier, 1996 : 227, 233234.

I-1-52 La proposition enrage les habitants d'Abuala :

- Nous trouvons que dix brasses de monnaie de coquillages et mille dents, c'est gros ; dix ignames pour dix brasses de monnaie de coquillages et mille dents, c'est gros.

Alors, néanmoins, on fait l'offre à d'autres villages.

Tous situés, comme Abuala, à Petite Malaita. Il s'agit de la constitution d'un premier réseau de prise de connaissance de cette nouvelle chose, l'igname, réseau de villages qui vont la rejeter.

I-1-53 Tous ces villages réagissent de la même manière :

- Dix choses comme ça, dont on ne sait pas si elles vivront ou mourront, c'est gaspiller notre argent.

Ils enragent :

- Levons une armée et massacrons les gens d'Alai avec leurs ignames.

Ils lèvent donc une armée de mille hommes, et disent :

- Allons les massacrer, ces gens d'Alai.

I-1-54 Les deux Faneakwaakwa ont vent de la chose

Informés par l'esprit de leur clan.

et disent :- Prenons la fuite.

I-1-55 Ils partent avec l'igname, et ils viennent vers ici avec Maioa, Sialage, Lofanikwala, Kwatoro, Fuanukunuku, Fuaamara, les deux Faneakwaakwa, ils prennent une barque et ils y chargent l'igname.

Ethno-étymologie de ces noms : Maioa, voir ci-dessus, I-1-37; Sialage $=s i+$ alage $«$ dans la direction/pour» + «sorte d'algue»; Lofanikwala $=l o f a+n i+k w a l a ~ «$ huile de noix de coco » + particule du génitif + " génération », c'est-à-dire "procréation et descendance»; Kwatoro $=k w a t o+$ oro " bâton fouisseur à ignames » + «nombreux »; Fuanukunuku $=$ fua + $n u k u$ 《fruit/graine/semence » + «proliférant/ fourni »(redoublé ici); Fuaamara $=$ fua + amara « fruit/graine/semence » + « stérile ».

I-1-56 Et ils traversent le détroit entre les deux Malaita et ils abordent la pointe méridionale de Grande Malaita.

I-1-57 Les deux Faneakwaakwa disent :

- Cherchons un village dominant la mer et à huit collines, et nous nous y établirons. Ils doublent la pointe, remontent vers le nord.
« Huit », à Malaita comme ailleurs en Océanie, est un chiffre parfait. Un commentateur ajouta, après avoir entendu cette version, que le choix du site par les émigrés n'était commandé que par la psychologie du narrateur : « Il fallait, pour lui [le narrateur] que l'emplacement fût bien en vue de sorte que tous ceux qui le doubleraient [dans leurs pirogues] le vissent bien ». Selon d'autres commentateurs, eux aussi spécialistes de leurs traditions, il fallait un site à huit collines parce qu'il existe huit variétés d'ignames et, surtout, un site bien irrigué qui reproduisît les conditions écologiques de la niche originelle du tubercule.

I-1-58 L'armée part en campagne et investit Alai mais trouve la place vide, tout le monde ayant fui dans la barque.

I-1-59 Là donc, cette igname, elle est bien comme un homme: si on dit d'elle qu'elle meure, elle meurt ; si on dit d'elle qu'elle vive, elle vit.

Cette réflexion insérée par le narrateur s'inscrit dans le contexte du massacre des humains et des ignames projeté par l'armée des villages en colère. Elle a aussi trait, selon un commentateur, à la nature dangereuse de l'igname, qui, en ce temps-là, n'obéissait qu'aux deux Faneakwaakwa. À Bae, l'igname parle aux hommes. L'espèce dangereuse donne des instructions à ceux qui la récoltent. À cause de la crainte qu'inspirait cette espèce inquiétante, on la renvoya à sa contrée d'origine.

I-1-60 La barque poursuit son voyage le long de la côte de Malaita. On double plusieurs lieux dont aucun n'est jugé convenable, faute de huit collines.

I-1-61 Enfin, on aperçoit Bae'ilangi

Bae'i langi : $i$ langi signifie " en hauteur »; bae, polysémique, signifie aussi bien "sanctuaire » que « parole » mais aussi « tuteur pour plants d'ignames »; ce site se caractérise par huit collines en hémicycle, derrière l'îlot Fuaga (voir carte 2).

et les deux Faneakwaakwa disent :

- C'est là que nous nous établirons, en cet endroit que nous voyons.

I-1-62 [Suit la description de la manœuvre d'entrée dans la lagune par la passe dans le récif.]

\section{I-1-63 Maioa dit :}

- Mes deux hommes, les deux Faneakwaakwa, ils sont venus ici, ils abordent à cet endroit nommé Areko.

Arekolarego " espèce d'arbre à pain, Garinga floribunda» (voir ci-dessus dans l'introduction, 
sur l'arbre à pain dans le panneau gravé de Barrau), dont on mélange la sève laiteuse à du charbon de bois du même arbre, réduit en poudre et macéré vingt-quatre heures dans de l'eau de mer. On stocke cette peinture noire (bai) dans des tubes de bambou, l'applique ensuite sur les dents pour les noircir de façon permanente. La feuille de l'areko maintient le produit en place pendant les dix jours de réclusion que dure ce rituel important (oogo), dix jours de réclusion dans une hutte spéciale, durant lesquels on ne peut s'alimenter que de liquide au moyen d'un chalumeau de bambou.

\section{I-1-64 Là donc, les habitants de Maelana'usu}

Village situé sur la côte, dominant l'îlot Fuaga ; voir ci-dessous, I-1-68. Quant aux autres noms de lieux, il s'agit de villages dans le voisinage de Fuaga.

voient arriver cette barque et ils se demandent d'où elle vient.

- Si c'était une embarcation de Gounaikwai, nous le saurions ; sinon, si c'était une embarcation de Leili, nous le saurions ; sinon, si c'était une embarcation de Uru, nous le saurions; sinon, si c'était une embarcation de Ngwangwasila, nous le saurions. Cette embarcation, c'est une embarcation d'un village différent.

Et ils sont là comme ce promontoire, dont le nom est Maelana'usu, et ils regardent et ils voient cette embarcation atterrir.

I-1-65 Les nobles Taalau, Bibirakwao, Aiofe, Aboa

Les nobles du village de Maelana'usu. Ethnoétymologie des noms: Taalau = taa + lau «quoi » + « encore»; Bibirakwao = bibira + kwao "pousse/rejeton/plante grimpante + blanc" (comme l'igname); Aiofe $=a i+o f e$ " plante »+ « don gratuit, désintéressé »; Aboa « anxieux ».

descendent au débarcadère et les habitants des villages voisins [dont la liste est donnée] se rassemblent pour regarder cette arrivée. C'est une très belle barque, elle ressemble au barukwaoa.

Barque typique des Salomon. Les Lau ne construisent un barukwaoa (baru «barque, grande embarcation »; kwaoa « blanc », à cause des plumes de mouettes qui en ornent la proue et la poupe surélevées) que pour un grand rituel, une fois environ tous les vingt ans, dans le cycle des cérémonies funéraires. Les grands-prêtres en supervisent l'exécution, qui dure quelque six mois, par un collectif d'hommes astreints à des tabous spécifiques. On le fabrique en planches qu'on colmate, l'incruste de nacre et de disques de conques (Strombus), le pare de plumes de mouettes et de calao. Les incrustations ornent flancs et extrémités, les unes géométriques, les autres représentant des bonites et des aiglesfrégates aux ailes déployées. Le barukwaoa ne prend la mer que pendant dix jours : son équipage, sujet à des contraintes rituelles importantes, le fait admirer par d'autres clans que le leur qui lui offrent des prestations. Après quoi on hisse la barque dans l'aire des hommes et l'expose aux intempéries pour qu'elle se dégrade le plus rapidement possible. Dès que le processus de dégradation commence, on met en route la préparation de la prochaine grande phase rituelle.

\section{I-1-66 Et ils demandent :}

— Vous êtes des gens d'où?

Les deux Faneakwaakwa disent :

- Nous sommes des gens d'Alai [suivent les autres noms d'Alai] et nous venons avec l'igname. Les gens de Mamalau [suivent les noms des autres villages] ont levé une armée de mille hommes et ils ont brûlé Alai après notre fuite.

I-1-67 Là donc, Bibirakwao, Aiofe, Aboa mènent leurs gens et ils montent à Maelana'usu.

I-1-68 Ils arrivent et ils prennent cette igname et ils la plantent en cet endroit appelé «la fondation de l'igname à Maelana'usu ».

«Fondation» parce qu'on y implanta l'igname pour la première fois à Malaita et aussi parce que accompagnèrent cette implantation la mise en œuvre des rituels de guérison des maux (mortels) entraînés par la violation de tabous reliés à l'igname. L'étymologie de Maelana'usu éclaire aussi la parenthèse qui suit dans le récit : maelana + 'usu: maelana "cérémonie funéraire»; 'usu « annuler, repousser, effacer». Maelana'usu tirerait peut-être son nom des pouvoirs de correction par neutralisation des conséquences néfastes de mauvais comportements envers le tubercule ; voir ci-dessous, I-1-70, I-191.

I-1-69 Si un homme vole quelque chose et qu'il le nie par la fondation de l'igname, il meurt, et il ne vit pas. On a donc appelé ce lieu la « fondation de l'igname », à Maelana'usu.

I-1-70 Là donc, ils démarquent ces deux terrains appelés Kwarosakae et Gwefufuri.

Dialecte baegu, les Baegu habitant le grande île face aux Lau, au haut de la partie méridionale de la lagune. Le narrateur donne comme glose lau de Kwarosakae 'umari lea kou qui signifie, 'umari «l'écaille de l'abalone/ormeau à lèvres noires » utilisée pour détailler en découpes les ignames qu'on va planter; et cette écaille lea kou 
« s'en va ». Quant à Gwefufuri, il s'agit, gwe, de la qualité (de l'écorce) de l'arbre fufiri, utilisée dans le traitement de la maladie causée par des impropriétés de conduite envers l'igname, c'està-dire une enflure de tout le corps qui provoque un décès rapide ; le même traitement vaut contre toute enflure et surtout contre celle causée, à l'instar de l'igname maltraitée, par celle du monstre de la mer, l'agaloniasi (voir aussi infra, I-1-93).

I-1-71 Ils les démarquent et ils les aménagent. Ensuite, ils les enclosent, ils les fouissent au bâton, ils plantent 20000 découpures [d'ignames]. L'esprit de l'igname continue, la fait proliférer (gulufa).

I-1-72 Là donc, ces deux plantations, on y met des tuteurs et l'igname devient bien fournie.

I-1-73 Ces nobles, Faneakwaakwa et Maioa, ils enseignent le rituel de la plantation de l'igname, et le pouvoir à cette fin, le pouvoir de cette plantation.

I-1-74 Ce noble, Maioa, il dit là-dessus :

- Cette plantation est presque mûre.

Et ils prennent une plante appelée « plante de conception », et ils prennent une deuxième plante, la «plante pour faire profiter » et ils prennent une troisième plante, la « plante pour enraciner $»$. Ils prennent une quatrième plante, la « plante pour faire mûrir ».

Il s'agit de quatre plantes dont on dispose des feuilles dans les sanctuaires au centre de la plantation (suumabo) à intervalles d'une ou deux semaines selon les stages de croissance de l'igname.

I-1-75 Voilà donc. La plantation mûrit.

I-1-76 Tous ces villages lau [énumération de six villages] se rassemblent devant ces deux plantations.

Ces lieux forment le premier réseau « positif » de distribution de l'igname, en contraste avec celui du rejet (ci-dessus, I-1-52). Pour le second réseau, voir I-1-82.

I-1-77 Les deux Faneakwaakwa dispensent leur enseignement.

I-1-78 Voilà la plantation presque mûre, on a pris ces quatre plantes, on les a prises et on a accompli tout le rite de la croissance de l'igname et on a libéré l'igname.

Faamola « neutraliser/libérer ». A cette fin, on remet au prêtre des feuilles fanées de plants d'igname pour qu'il effectue le rite de asikwaikwai (asi « tomber »; kwaikwai « ensemble », allusion au fait que l'igname mûre perd ses feuilles d'un coup ou presque). Le prêtre pro- nonce alors une invocation aux esprits à la suite de quoi l'igname peut être récoltée.

I-1-79 Cette plantation a atteint la maturité, toutes les feuilles sont parties, tombées; ils retirent les plantes à vertu multiplicatrice, et ils récoltent les tubercules de ces plantations.

I-1-80 Ils déposent le pouvoir de prolifération (gulufa) dans les deux abris d'ignames et ils déterrent les tubercules.

Abris : cases à même le sol où on entrepose les ignames, juste à côté de la plantation. Y dorment les hommes qui travaillent à la récolte et dont la condition demeure tabou tant que dure leur travail.

I-1-81 De ces deux plantations, ils récoltent 20000 ignames.

I-1-82 Les gens de Maana'oba [etc. : énumération de treize villages de Malaita septentrionale] s'assemblent devant les deux plantations, et on leur partage les ignames.

Second réseau de distribution, $c f$. I-1-76.

I-1-83 On prend deux cents ignames et c'est la part de Maana'oba et ainsi de suite [passage répété pour cinq autres villages lau]. Chaque village reçoit une part de deux cents ignames pour les planter à leur tour. Et on leur enseigne également comment faire les plantations et on leur enseigne aussi quelles plantes [magiques] on doit utiliser pour les plantations.

I-1-84 Le noble Taalau, chef de Maelana'usu, s'adressa en ces termes aux Lau :

- Vous emportez avec vous cette igname; plantez-la et donnez des fêtes de l'igname.

Maoma "grande fête cérémonielle": nom générique des phases majeures - de huit à onze selon les clans - du cycle rituel le plus important, suivant la mort d'un grand-prêtre et s'étendant sur une vingtaine d'années. On groupe ces phases en "cuites » et "crues » selon qu'on y offre des aliments cuits au four de terre igname, taro et la viande de porc - ou crus, c'est-à-dire exclusivement de l'igname, du taro et des noix canarium fumées dans des bambous. Lors de l'avant-dernière de ces fêtes, on construit le barukwaoa (supra, I-1-65).

I-1-85 «Que les femmes n'installent pas de tuteurs. Si une femme le fait, il lui est interdit de traverser le maanabisi, et elle mourra ».

Maanabisi : l'aire des femmes et où se trouvent les quartiers d'une part des femmes en règles, de l'autre, des femmes en couches, et où les unes et les autres doivent impérativement séjourner, selon leur état ; là se trouvent aussi leurs latrines. Or si une femme installait des tuteurs, elle 
«serait faite homme», faangwane (faa préfixe causatif + ngwane homme) Entrerait-elle dans le quartier des femmes, elle subirait alors le même châtiment qu'un homme : la mort immédiate.

I-1-86 Aujourd'hui, même les femmes chrétiennes ne posent pas de tuteurs à ignames. Les femmes en état de bisi [menstruées] ne mangent pas d'ignames de nos jours non plus. Même aujourd'hui, les femmes au bisi ne mangent pas d'ignames.

Ramoagalo insiste : même dans le contexte actuel, malgré l'influence des missions.

I-1-87 Aux premiers temps, l'igname était vraiment tabou. Si un homme dormait dans sa case familiale, il ne pouvait entrer dans sa plantation.

On traite, avec un certain mépris, de boko $i$ luma (bloqué + dans + maison familiale) l'homme qui passe la nuit dans sa case familiale, dans l'aire mixte des îlots, au lieu de dormir dans le beu de sa lignée dans l'aire des hommes. Par implication, on entend que cet homme aura eu des relations sexuelles avec sa femme.

I-1-88 De même, un homme qui avait mangé du lakeno ne pouvait pas, non plus, entrer dans sa plantation.

Lakeno " gâteau fait de taro et de noix de coco pilés ".

I-1-89 Si une femme enjambait une igname, elle en mourrait. Il n'y a que les hommes de Maelana'usu qui pourraient sauver cette femme d'une mort certaine, nul autre homme.

Enjamber - littéralement, " vaginer »- une partie du corps de quelqu'un de l'autre sexe ou même un objet lui appartenant, viole un tabou imposé rigoureusement et sous des peines sévères, aux hommes comme aux femmes. On verra d'ailleurs (I-1-92, 93) que les tabous de l'igname visent l'homme tout autant que la femme.

I-1-90 Ils la sauveraient au moyen d'une plante appelée sarufi, elle pousse dans la terre.

Sarufi « Litsea domarensis ». Haru/saru « pustulence »; fii « faire mal ».

I-1-91 Si une femme enjambe une igname, elle enfle ; le noble Daui saura cependant la guérir, il connaît de nombreuses plantes puissantes, il la guérira selon la méthode de l'igname de Maelana'usu - pas selon une autre méthode.

Daui, frère aîné du narrateur, présent lors de la narration comme mentionné en introduction à cette variante, prêtre de lignée, "savant» ngwane liotoo - et grand spécialiste des cures difficiles. Il a obtenu ce pouvoir et d'autres soit de son père, soit de son oncle maternel, soit en les achetant à leurs détenteurs.

I-1-92 La méthode de l'igname de Maelana'usu, qu'un homme en applique la puissance et la femme guérira, ou, si c'est le cas, un homme.

I-1-93 Et il est tabou de piler des tubercules d'ignames dans un bol comme le tien. Si un homme en mange [de l'igname pilée], il mourra. L'igname le fera enfler et il ne pourra plus dormir. Son estomac grossira comme ça.

Le narrateur désigna un bol dans notre case. Un commentateur cita le cas d'un homme décédé quelques mois auparavant parce qu'il en avait pilé ; il ne put être sauvé parce que, chrétien, les esprits le considéraient comme contaminé de toute façon et ne daignèrent pas le guérir. L'enflure - souvent dite mortelle — résulte de violations de tabous en maintes cultures océaniennes. Pour un témoignage, $c f$. Granada Television (1987) où un prêtre affirme que la transgression d'un tabou - entrer dans la case d'un grand-prêtre décédé sans en avoir le mandat - ferait enfler démesurément le pénis d'un homme « qui deviendrait aussi gros que le tien » (parlant du pénis de l'anthropologue).

I-1-94 L'origine de l'igname à Alai, je l'ai donc racontée.

En fait, il s'agit de l'origine de l'igname également à Malaita, où on l'implante en territoire baegu (Baeilangi) mais étroitement associé à Fuaga, en territoire lau dans la lagune, comme tous les villages auxquels on distribue gratuitement l'igname.

Là s'arrête formellement, par cette formule qui termine habituellement tous les récits, la variante de Ramoagalo. Il n'en ajoutera pas moins les commentaires suivants.

\section{I-1-95 La mère de l'igname s'appelle reetaba.}

Le terme "mère » désigne en lau ce que la botanique occidentale nomme "genre» ou «primary taxon». On ne la gardera pas à Malaita où on ne plantera que sa "progéniture », c'est-à-dire les espèces énumérées ci-dessous (I-1-97) et plantées sur les huit collines de Bae-i-langi.

I-1-96 Si on la racle à Uongwane, on entend le bruit. Les gens de Maelana'usu disent :

- Nous raclons la reetaba, et on l'entend fort et c'est pourquoi nous disons que cette igname s'appelle reetaba, qui a été renvoyée à Alai [suivent les autres noms d'Alai]. Si nous raclons cette igname, il n'y a pas un homme, si loin soit-il, qui n'entende le bruit. Cette igname-là, la reetaba, nous l'avons renvoyée parce que le bruit 
était trop fort. Nous n'avons gardé que l'igname normale.

Cette igname «bruyante, indiscrète » a été renvoyée, surenchérit un commentateur, parce qu'elle compromettait la privauté des habitants de Maalana'usu : ils ne pouvaient en apprêter sans que tout l'univers ne le sût. Voir aussi ci-dessus, I-1-59. Selon une autre variante (I-9), cette mauvaise igname - cannibale par surcroît — et qu'on rejette, s'appelle kou, i.e., « dehors!, va-t'en!».

I-1-97 Les espèces en sont daungwao, kooru, luka, dikare, logea, losu, dikare abu, kaifioemanu; ces espèces sont demeurées parmi nous.

Le narrateur caractérisa chaque espèce comme suit en réponse à une question : daungwao : dau + ngwao «tenir ferme» + « ficelle végétale »; référence au fait qu'on attache fermement à son tuteur cette igname haute à tubercule blanc; kooru tubercule rond et gros; luka tubercule rond, aplati, dur sous la dent, d'où (?) son nom dérivé du verbe luka "abandonner, rejeter »; dikare semblable à la daungwao mais plus petite ; logea assez haute, à tubercule blanc ; losu tubercule courbe qui évoque un serpent, pelure rouge, pulpe blanche; dikare abu: dikare $+a b u=$ "sang ", dikare couleur de sang; kaifioemanu: $k a i+f i o+e+$ manu: « igname» + «peler» + copule + « oiseau»: mince, encore plus grande que la daungwao, tubercule rouge.

I-1-98 La reetaba a été renvoyée, elle n'est pas demeurée à Malaita.

Toutes ces autres ignames sont restées chez les Lau. Les gens de la terre ne cultivent pas l'igname. Il n'y a que les gens de la mer, appelés Lau, qui cultivent l'igname; à l'intérieur, non.

Toutefois, le narrateur ajouta, après coup : « Mais aujourd'hui, quelques-uns la cultivent à l'intérieur ». En fait, plusieurs des villages nommés comme récipiendaires d'ignames lors de la distribution suivant la première récolte, sont des villages de l'intérieur mais situés à une distance assez courte (1 ou 2 heures de marche) de la côte.

A une question, Ramoagalo répondit que le taro, comme le feu, précédèrent de beaucoup l'igname à Malaita ${ }^{3}$.

\section{Sommaire de données ethnographiques pertinentes}

Je retiens comme critère de pertinence pour présenter les données ethnographiques qui suivent, leur rapport à la transformation de l'igname en poisson et son rétablissement par renversement de la métamorphose. Je justifie ce critère dans une double perspective, une première, spécifique et plus étroite, une seconde, holiste et plus vaste. En effet, quant à la première perspective, je signale que j'ai obtenu des variantes du même mythe tout aussi bien à partir d'enquêtes sur le sens du poisson alia que sur celui de l'igname. Quant à la seconde perspective, on ne peut contextualiser le mythe qu'à l'intérieur de synergies fondamentales à Malaita : les synergies terre-mer-femme-homme comme nous le verrons maintenant. (On trouvera des développements de la plupart des données ci-dessous dans Maranda et Köngäs Maranda 1970 et Maranda 1979, 1982a, 1985, 1997, 2001.)

(1) Métamorphose de poissons en légumes sur les marchés. L'institution des marchés remonte aussi loin dans le passé que l'origine des îles artificielles. Sur ces places côtières, "gens de la terre » et « gens de la mer» se retrouvent pour échanger une fois la semaine - en fait tous les cinq jours en accord avec le mode de décompte des Malaitains qui regroupent les jours en unités de cinq selon les avant-veilles, veilles, jours de marché et leurs lendemains et surlendemains. Les femmes occupent les centres - en cuvettes - des places de marché, sur les rebords desquels se tiennent d'un côté les " hommes de la terre », de l'autre, ceux « de la mer».

Là, les femmes lau apportent les poissons pris par leurs hommes (pères ou frères classificatoires, maris) et les troquent ou les vendent contre les légumes en provenance des jardins des vis-àvis de la lagune et qu'y ont apportés leurs homologues « de la terre ».

(2) Initiation des jeunes femmes : rituel de nubilité. Lors de ses premières menstrues, la jeune femme lau passe le temps prescrit dans le quartier des femmes. On marque son retour au quartier mixte et son entrée en nubilité par un rituel aussi simple que significatif. Elle et d'autres jeunes femmes devenues récemment nubiles forment une ligne : assises sur le sol, avec, de part et

3. Il faudrait traiter du taro et de l'igname en complémentarité, l'un substitut de l'autre dans nombre de contextes mélanésiens ( $c f$. Barrau 1956 : 72-86). Voir par exemple, pour une pratique inversement symétrique à celle des Lau, F. Panoff $(1969: 22)$ « The Maenge have special mamisa [lesser yam] gardens which provide food for the wet season. They perform very little magic in these gardens, whereas taro gardens are the focus of elaborate rites ". Par ailleurs, $c f$. M. Panoff (1970b: 240), " The taboos are stricter as regards the yam gardens than the taro gardens ». Cf. aussi Bonnemaison $1996: 262 \mathrm{ss}$. 
d'autre, formant deux lignes, d'un côté des légumes (de préférence des ignames) et de l'autre, des poissons. Tout en chantant une mélopée fermement rythmée, ces jeunes femmes prennent un légume dans une main, un poisson dans l'autre et, croisant les bras, les permutent. Ensuite, elles les consomment dans un repas cérémoniel. Ce rituel les habilite à jouer leur rôle de femmes sur les marchés, opératrices métamorphosant les fruits de la mer en ceux de la terre quant aux lau, ceux de la terre en ceux de la mer quant aux montagnardes.

(3) Initiation des jeunes hommes: rituel de nubilité. Si la jeune fille devient spontanément nubile lors de ses premières menstrues par l'écoulement de sang qui la montre capable de donner la vie, le jeune homme, lui, ne deviendra homologué qu'après une opération qui le rend comparable à sa contrepartie. En effet, il devra faire couler le sang d'un ennemi, se montrant par là capable de donner la mort. Et il fera cet exploit en utilisant l'arme par excellence, le " clitoris ", une massue représentant sous forme de losange un clitoris en érection, ou encore un casse-tête dont le sommet s'orne de deux clitoris en érection.

(4) Structure des îles artificielles. Chaque île artificielle se divise en trois aires : mixte, où on trouve les cases familiales; féminine, interdite aux hommes; et masculine, interdite aux femmes. L'aire des femmes comporte comme son centre et endroit le plus " chargé » la case des couches où la mère doit demeurer avec son bébé durant les trente jours suivant l'accouchement. Les autres cases reçoivent les femmes menstruées. Le centre et endroit le plus chargé de l'aire des hommes tire son sens de la fosse aux crânes qui l'occupe. Là, le grand-prêtre dépose, pour leur donner naissance au monde des esprits, les crânes des membres de son clan décédés depuis la mort de son propre père. Les autres cases servent de club-houses et dortoirs aux hommes, où ils doivent demeurer en réclusion chaque fois que se prépare une phase de rituel collectif. Et, lors du décès d'un parent, un de ses proches devra demeurer trente jours en réclusion dans l'aire des hommes.

(5) Structures de production. Comme partout en Océanie, le haut appartient aux hommes, le bas, aux femmes. Les implications de cette dichotomie importent quant à l'organisation du travail et de la vie. Ainsi, la lagune appartient aux hommes à marée haute ; ils y pratiquent évidemment la pêche, activité interdite aux femmes. Par contre, à marée basse, la lagune appartient aux femmes, qui y collectent mollusques et coquillages - aliments interdits aux hommes, en cela proscription sans sa symétrique puisque les femmes consomment librement de tout poisson (sauf certains, en grossesse).

\section{Analyse sommaire}

Dans cette esquisse d'analyse, je vais reprendre le mythe (variante I-1) en suivant son déroulement. J'ajouterai aux commentaires fournis en retrait et en italique au fil du récit pour les approfondir ou les déployer, afin de permettre au texte de donner le plus de rendement possible.

(1) Départ des héros et séjour dans la terre d'origine de l'igname (I-1-1 à 7). Une autre variante, racontée par le grand-prêtre de l'igname à Fuaga ( $c f$. Cartes 1 et 2), dit comment leur père les envoie dans la terre des goosile, monstres cannibales, pour aller y chercher l'igname. L'aîné, fait prisonnier et fumé par les monstres qui s'apprêtent à s'en régaler, puis secouru par son cadet, vient avec son aide à bout des monstres. En récompense pour ce haut-fait, les habitants de la terre d'origine de l'igname leur donnent un finisi (" prix du sang »). Les frères refusent toutes les prestations monétaires, se contentant d'accepter huit ignames et deux jeunes femmes.

(2) Noms propres de lieux (I-1-2). Le pays des goosile porte le nom de Uluulumalau (ulu " patauger» ici redoublé + malau « [dans les] profondeurs »), un des noms lau de Maramasike, ou, dans une autre variante, on l'appelle Ulunimatakwa : ulu $+n i+$ matakwa $=$ " patauger $»[$ to wade], + « dans » + " profondeurs marines ».

Sans vouloir ajouter ici au grand nombre de traités de toponymie, je commenterai brièvement les noms de lieux dans le mythe qui nous occupe. L'énumération des noms de ces lieux qu'on double en mer ou où on aborde, ou encore de ceux qu'on traverse lorsqu'on fait le trajet par voie terrestre, revient constamment dans les récits lau - comme d'ailleurs dans maints rituels - , et on reconnaît un diseur compétent par l'assurance et l'exactitude avec lesquelles il les cite tous.

Notons d'abord que cette topographie sert en quelque sorte de "cartographie cognitive» ou d'aide-mémoire. En effet, un diseur chevronné à qui on demande s'il peut raconter tel ou tel mythe esquissera souvent dans l'espace, sans paroles, avec l'index de sa droite, une cartographie des trajets de l'action, des parcours des personnages. Si son geste reste suspendu, il 
répondra qu'il ne connaît pas ce mythe suffisamment bien pour le raconter. Si, par contre, il achève son geste, il s'exécutera.

En outre, les mythes se trouvent ainsi, par le répertoire des noms de lieux qu'ils contiennent, à enseigner la géographie aux enfants. A preuve : un jour, une jeune femme nous accompagnait en mer hors de la lagune dont elle n'était encore jamais sortie. Elle n'en «reconnaissait» pas moins les pointes, promontoires et autres repères topographiques que nous doublions. Elle expliqua que cette connaissance lui venait des mythes entendus sur la place du village. A preuve encore : le diseur d'I-1, Ramoagalo, nous fournit une description fort détaillée et précise de l'atoll d'Ontong Java (Suu-i-Robo pour les Lau). Il en nomma les terres, les récifs, les passes dans le récif, etc. Peu après, sur une carte de cet atoll, je pus constater l'exactitude de sa connaissance de la géographie des lieux - tenant compte de variations de type glottochronologique. Or Ramoagalo n'avait jamais vu Ontong Java : la connaissance qu'il en avait lui provenait du mythe d'origine des dents de dauphin que des immigrants à Malaita ont apportées de cet atoll.

De fait, et comme le souligne Hviding (1992 : 252 ss ; $c f$. aussi Malinowski 1922, 1935, sur la signification des cartes du kula), la cartographie mentale permet la construction non seulement d'une connaissance géographique - fort vaste, chez les Lau, puisque leur ethnogéographie comporte plusieurs îles d'archipels différents - mais aussi celle d'une identité. Le réseau des noms de lieux fournit en effet une emprise cognitive sur un environnement à la fois religieux, géopolitique, socio-culturel somme toute, qui garde vive la connaissance d'un univers englobant aussi bien des lieux mythiques que physiques et qui témoigne de leurs sens historiques. A cet égard, on peut relever une corrélation entre le nombre de noms que porte un lieu chez les Lau et sa charge d'histoire. Pouvant atteindre une dizaine, ces noms - dont des diseurs compétents ne manquent jamais d'énoncer la liste complète lors de chaque narration - constituent une sorte de précis de l'histoire locale. On peut en voir quelque peu la portée à propos de la constitution de réseaux de rejet de l'igname (I-1-52) et de ceux de son appropriation (I-1-76).

Reliées aux grandes gestes des héros culturels dont les cycles narratifs les parcourent, de quêtes en quêtes, d'exploits en exploits, ces toponymies convoquent et dynamisent des sites dont une cartographie profonde reste à faire ( $c f$. Hviding 1992, ch. 6).

Les noms de lieux reçoivent aussi leurs investissements sémantiques par leurs habitants, dont le récit nomme généralement les principaux (nobles) chaque fois qu'il mentionne un lieu. Ainsi peuplés, les noms de lieux prennent dénotation et résonance qui en déploient le sens. (Cf. Hviding (1992 : 263, 282 ; Maranda 2002) reprenant la distinction de Parmentier entre « signs of vs in history ».)

(3) Noms propres de personnes (I-1-6). On a pu montrer que les noms propres de héros offrent souvent un résumé fort concis de tout un mythe (Lévi-Strauss, 1966 ; Hymes, 1972 ; Maranda 1970). Cela vaudrait-il pour le nom des héros de notre mythe? J'en ai mentionné l'ethnoétymologie à propos de I-1-6 mais je reviens sur la question pour rendre compte d'un débat avec des philologues lau à ce sujet.

J'ai donc discuté avec plusieurs informateurs l'ethno-étymologie de Faneakwaakwa, le nom des héros, à partir de l'hypothèse qu'on pourrait prendre fane dans son acception de "igname sauvage », conservant celle de " mud hopper» — la seule possible - pour akwaakwa. J'ai formulé cette hypothèse en utilisant l'argument que le rapprochement entre l'igname sauvage, qui pousse sur la berge dans la boue, et un poisson qui se meut dans la boue à la pointe des marées, conviendrait comme nom aux héros qui vont apporter à Malaita l'igname cultivée après sa transformation en un poisson pêché par la suite. Bien que reçue comme logique et pertinente, tous les philologues lau rejetèrent systématiquement cette interprétation, sauf Ramoagalo, le narrateur de la variante I-1.

(4) Mode d'appropriation de l'igname (I-1-7). La mythologie nous apprend que des Lau procédèrent aussi par vol pour s'approprier un autre tubercule, la patate. Dans ce cas, suite à l'interdit d'en rapporter des crues à Malaita, et pour déjouer les inspections auxquelles on soumit les paniers et pirogues lors de leur départ, les héros en dissimulèrent des découpures sous leurs prépuces. Je rappelle aussi que dans la variante du grand-prêtre de l'igname (supra, à propos de I-1-1 à 7), les héros, au lieu d'en voler, obtiennent huit ignames en récompense pour leur exploit.

(5) Naufrage des héros (I-1-9). Il s'agit dans cette variante comme dans la plupart des autres d'une tentative de neutralisation du vol de l'igname. A cette fin l'esprit utilise une tempête ( $c f$. la tempête déchaînée par Junon contre Énéée dans L'Énéide, chant 1). Dans la variante du grand-prêtre de l'igname, les héros ayant reçu les tubercules en récompense, le mythe doit trouver une autre raison à leur échec : ce sera la présence 
de femmes dans la même embarcation que l'igname qui la fera chavirer.

(6) Sommeil des héros (I-1-16 à 26). Dans cette variante, le poisson souffle sur les yeux des pêcheurs : dans les rituels lau, le souffle sert de contact subtil entre l'officiant et l'objet à consacrer, entre le guérisseur et le malade. Le poisson se comporte donc comme doué de ce type de pouvoirs, qu'il utilise pour rendre inefficace l'effort des héros. On aurait là une ultime tentative de neutralisation de l'appropriation de l'igname par les Malaitains. Dans I-1, l'astuce des héros déjouera la manœuvre du poisson; dans d'autres variantes, l'esprit de leur clan viendra à leur aide pour les garder éveillés : esprit contre esprit..., celui du clan des héros s'avérant vainqueur.

Le grand-prêtre de l'igname dira, en réponse à ma question après son récit, que ce pouvoir surnaturel de l'igname lui venait de ce qu'elle était un esprit - pas une véritable igname qu'on pourrait manger, ajouta un commentateur ( $c f$. I-1-35). Ce dernier passa en revue les métamorphoses de l'igname de la façon suivante : l'esprit se transforma en igname, l'igname en poisson, le poisson de nouveau en esprit, ensuite l'igname persista sous forme de poisson, ce poisson se transforma en igname et aborda (fut pêché), et ses écailles se métamorphosèrent en igname.

Un autre narrateur, un chrétien, attribuera le sommeil des héros à une cause "naturelle»: leurs yeux fatigués par une trop grande concentration du regard sur l'eau.

(7) Capture et espèce du poisson (I-1-14, 27). Il existe une ressemblance entre l'unugwaila et l'alia.

"Hump-headed Maori wrasse... One of the most beautiful labroids, and also the largest, in the IndoPacific reg... Large examples develop a big fleshy crest or hump on the head above and in advance of the eye, somewhat similar to that of the Snapper, Chrysophrys auratus. A large fish caught at Hayman Island, Whitsunday Group, North Queensland, and called a Bluetooth Groper was probably this species. It measured 7 feet 6 inches in length and weighed 420 pounds.

On the Queensland coast north of Bundaberg the species is commonly caught [comme à Malaita où nous en avons vu pêchés] in the deeper waters of the reefs along the edges of the coral shelf. " (Marshall 1964 : 315).

Somme toute, il s'agit d'un poisson de haute mer - par opposition à un poisson de lagune à écailles larges. Cependant, ni les autres pêcheurs, ni même le narrateur, plusieurs années plus tard comme mentionné ci-dessus, n'acceptèrent que ce poisson fût un unugwaila : tous s'accordèrent sur l'alia. On en donna comme raison la forme des écailles de ce dernier. Plus larges, en effet, que celles de l'unugwaila, elles ressemblent aux découpures d'igname qu'on plante en début de saison. (Cf. I-7-6, où, comme dans d'autres variantes, l'écaille ou l'igname, douée de parole, donne des instructions aux humains.) Un autre cultigène, le taro - que les Lau disent arrivé à Malaita bien avant l'igname - , doué de parole lui aussi, enseigna à une vieille femme la technique de la vrille à feu pour qu'elle sache le faire cuire.

Lors de la narration du mythe par le grandprêtre de l'igname, un auditeur intervint pour signaler qu'il avait vu l'endroit précis où les quatre frères avaient attrapé l'igname-poisson.

J'insère ici une donnée complémentaire sur l'igname-poisson, une " prière » en provenance d'Ulawa, terre d'origine de l'igname selon plusieurs variantes lau. De Coppet (1976: 170 citant Ivens 1927 : 335) l'introduit ainsi :

Le jardin est comme la surface de la mer : il cache les merveilleux poissons que sont les tubercules qui grossissent lentement dans la terre.

Une cordyline sacrée prend racine, une igname prend racine.

Toutes deux prennent racine dans mon jardin comme deux grands poissons des profondeurs qui ne peuvent s'échapper.

Comment les plantes prennent-elles racine?

Comme deux grands poissons des profondeurs elles se chauffent au soleil de mon jardin, commedeux baleines des profondeurs.

(8) Le goûteur d'igname (I-1-38 s.). Le chef du clan se risque à consommer de l'igname en premier et s'en trouve repu. Dans d'autres variantes, la première personne à manger de l'igname (suite à la famine qui dévaste son clan), entraînera une catastrophe parce qu'il n'a pas attendu les instructions de l'esprit sur le mode de traiter l'igname, et il sera banni de Maramasike.

Quelle que soit la variante, on trouvera une raison pour que l'igname arrive à Malaita. Ainsi, dans I-1-51 ss, le prix exorbitant qu'on demande pour l'igname causera le départ de ses porteurs sur Malaita.

(9) Tabous et prescriptions portant sur l'igname (I-1-41, 43, 78, 85 à 93, 96). Dans ce mythe-ci comme dans d'autres, une innovation importante suscite des inquiétudes quant à ses usages et maniements. Les Lau formulent donc des prescriptions, les unes relevant d'opérateurs culturels bien en place, d'autres, innovatrices - à propos desquelles on évoquera le pouvoir des esprits qu'on invoque - devant pouvoir gérer la nouveauté. Ici, on se rabat d'abord sur des opé- 
rateurs du premier type: il faudra offrir les prémices aux esprits, confier au grand-prêtre le soin rituel de bien effectuer la neutralisation (faamolaa: action par laquelle on fait passer un objet ou un comportement d'un état tabou à un état dont on a évacué le danger) ; on utilisera des tabous courants ou étendra leur portée, e. g., interdiction de contact avec les femmes en règles, interdiction pour un homme de dormir dans la case familiale et d'aller ensuite au falisi, etc.

Quant aux règles nouvelles et appropriées à l'élargissement de l'univers familier imposé par l'innovation, on procédera en partie par analogie. Ainsi, on installera, comme dans les jardins de taro, un sanctuaire au milieu de la plantation (suumabo) mais - ce qu'on ne fait pas pour le taro - on le garnira de plantes magiques particulières ( $c f$. infra, (12), Plantes magiques). De nos jours, dès le sol prêt pour la plantation, le prêtre s'adresse à l'igname dans ce suumabo, et il y plante les premières découpures d'ignames à aller en terre. Quand viendra la récolte, on commencera par les ignames du suumabo, prémices qu'on offrira aux esprits dans un sanctuaire situé à l'intérieur du beu du clan. En outre, on inventera des tabous distincts, comme celui de laisser choir, de piler ou de gratter l'igname.

(10) Falisi ( $I-1-48 s s)$. Nom de la plantation consacrée à la culture de l'igname, à l'exclusion de toute autre plante. Contrairement au taro, à la patate douce et aux autres cultigènes, qui peuvent partager un même jardin, on réserve toujours à l'igname une plantation exclusive. Pour les falisi consacrés aux grands rituels (maoma), on en aménage qu'un seul. Quant aux autres plantations d'ignames, les "profanes ", chaque famille peut faire la sienne. Les falisi cérémoniels se distinguent des autres en ce que, interdits aux femmes, on les enclôt d'une palissade imposante, avec des portes aux chambranles à longs poteaux sculptés représentant des ancêtres et, aux quatre coins, de grandes sculptures de requins et de mouettes. On associe par là l'igname d'une part à la mer par le requin et à l'air-mer par la mouette et, d'autre part, de façon plus explicite, aux esprits fondateurs du clan. En effet, l'ancêtre fondateur des Rere se métamorphosa en requin à sa mort - requin dont les parties du corps découpé rituellement et ensevelies dans différents sanctuaires de Malaita septentrionale construisirent un ferme réseau socio-culturel depuis Foueda jusque à la lagune de Langa Langa, face à Auki, sur la côte méridionale soit sur une distance de quelque quatre-vingtcinq kilomètres par bord de mer.
Cette alliance de type religieux-politique et consolidée par des mariages, se perpétue dans les "concessions" de récifs à coquillages recherchés pour la confection de monnaies que les Lau accordent aux lagunaires de Langa Langa. Les Langa Langa chez qui se trouve la frappe de la monnaie de Malaita viennent plonger deux fois l'an pour recueillir sur ces bancs les mollusques dont les lèvres pourpres fournissent les disques de plus grande valeur dans la confection des monnaies.

(11) Recherche d'un lieu approprié pour l'implantation de l'igname à Malaita (I-1-55 ss). Ramoagalo ne s'attarde pas sur le mode de recherche de lieux convenables par l'équipage en fuite et en quête d'un site pour l'igname. D'autres variantes, par contre, disent comment on a recours à la divination pour y arriver, ou encore comment l'homme chassé de son village par son fils se rend à Bae chez ses sœurs, mariées virilocalement comme le veut la coutume.

(12) Plantes magiques (I-1-74). On a là quatre plantes pour bien soutenir toutes les phases - de la germination naissance jusqu'au mûrissement — du cycle de vie de l'igname. Des commentateurs ont ajouté que les termes utilisés pour désigner les fonctions des deux premières plantes, à savoir de «faire concevoir» et de «faire profiter/engraisser », peuvent également désigner les étapes de croissance d'un bébé. Par contre, en réponse à une question, ils ont déclaré que ces mêmes plantes non seulement ne sauraient servir à assurer le développement sain d'un enfant mais que, fussent-elles essayées à cette fin ou à toute autre fin médicinale pour des humains, ceux-ci en mourraient. Ils insistèrent que l'analogie entre igname et bébé ne vaut que pour les phases de croissance ; cette analogie n'a donc pas de portée transversale. En outre, l'efficacité de ces plantes ne vaut pas pour d'autres cultigènes que l'igname.

(13) Noms des ignames (I-1-96). On peut noter ici une divergence entre ethno-philologie et " explication de texte». En effet, la leçon de I-1-96 justifie la motivation du nom reetaba par son caractère bruyant, indiscret, raison pour laquelle on la rejette et la renvoie à son lieu d'origine. "Nous raclons la reetaba, et on l'entend fort et c'est pourquoi nous disons que cette igname s'appelle reetaba, qui a été renvoyée à Alai ». Pour ce narrateur, reetaba signifie "source de vacarme». Mais des ethnophilologues lau fournissent des exégèses débordant cette explication. Je résume leurs commentaires. 
D'abord, les lexicographes lau rappellent que l'igname reetaba porte aussi deux autres noms : reetabailua et tabailua - auxquels on peut en ajouter un troisième (selon I-9-11), kou, qui signifie «dehors!»- et, exégètes, ils ajoutent que cette " mère ", i.e., forme originelle et genre de ses espèces, n'en reste pas moins fort dangereuse, voire une cannibale qui dévore les enfants si on la laisse seule en leur présence dans une case (selon I-9-11). Sa pulpe rouge lui vient d'ailleurs de ces repas d'ogresse.

Ensuite, les lexicographes fournissent des ethno-étymologies plausibles de ces trois vocables. (1) Pour reetaba: ree, terme réservé à la noblesse pour désigner l'action de manger; dans une de ses acceptions, taba signifie «détruire, enlever »; reetaba indiquerait donc la noncomestibilité, surtout pour les nobles, de cette igname mère. Disons en passant qu'aucun tabou n'interdît de consommer les "mères » d'autres espèces animales ou végétales. (2) Quant à tabailua, les Lau en proposent deux étymologies, chacune également valable selon eux : (a) taba « couper, trancher », employé ici au sens métaphorique ; $i$ particule locative ; lua «cou », ce qui donnerait « igname coupe-gorge », dans le sens d'étouffer, pour une nourriture trop amère ou trop sèche dit-on. Enfin (b) selon une autre de ses acceptions, taba pourrait, métaphoriquement encore, vouloir dire "s'étendre, se déployer » et, dans ce cas, i lua signifierait « dans la marée montante » ( $c f$. le nom des héros comportant akwakkwa, I-1-6). Le nom de l'igname mère renverrait peut-être ainsi à son arrivée à Malaita sous forme de poisson. Selon I-1-98, on renvoie la mère chez elle, ne gardant que sa progéniture ; selon d'autres variantes, on ne garda à Malaita que quatre des « enfants » de l'ignamemère.

Ces considérations et réflexions atteignent un niveau d'explication plus radical qu'une simple motivation de reetaba par le bruit que, raclée, l'igname émet. D'abord, mentionnons que seule l'exégèse (2) (b) n'aurait pas trait au rejet de reetaba par les Lau. Là, on retrouverait plutôt la métaphore métamorphisante (igname $=>$ poisson=>igname), formule générative du mythe. Par contre, les exégèses (1) et (2) (a), pertinentes quant au rejet de reetaba, en motivent davantage le nom. Elles se situent à un niveau sémantique plus profond, expriment une cause plus grave du rejet : le danger de mort que fait encourir l'association par contiguïté avec cette igname « coupegorge ».

\section{Interprétation}

La sémiotique a fait sienne l'anthropologie socio-culturelle (Maranda, 1982b). Elle a toutefois incité cette dernière discipline à porter plus d'attention à la signifiance, ou sémiosis, ce processus par lequel les signifiants deviennent porteurs de tels ou tels sens en même temps qu'ils les construisent. Je proposerai donc dans cette section une interprétation de ce type : comment prospecter la signifiance de l'igname chez les Lau de Malaita? Bien sûr, il s'agit de montrer comment son mythe d'origine non seulement s'inscrit dans une totalité mais aussi comment il la conforte.

Cet essai d'interprétation comprend quatre volets. (1) Je traiterai d'abord du mythe luimême en proposant une hypothèse sur sa genèse, sur l'opérateur de son architecture - hypothèse qu'on ne pourra cependant vérifier que, d'une part, par le consensus des Lau eux-mêmes et, d'autre part, par l'éclairage qu'elle peut projeter sur la société qui véhicule le mythe. La notion de métaphore telle qu'on peut la reformuler à partir des travaux de Hubert et Mauss (Mauss 1960 ; Maranda 1980) servira de point de départ à la formulation du constructivisme mis en œuvre dans le mythe. Ensuite, (2) j'élargirai le traitement au moyen de la notion de "métaphore métamorphisante » (Maranda 1993; 1997b) et de la résistance qu'oppose le « texte » d'une part (3) à la métamorphose et, d'autre part (4) à la consommation de l'igname. On verra ainsi comment le mythe "opère ", comment il s'irradie dans une contextualisation qui le façonne et que, en rétro-action, il consolide.

(1) Le processus métaphorique: Hubert et Mauss. Comme je l'ai montré ailleurs (Maranda, 1980), les trois opérations fondamentales de l'esprit humain mises en relief par Hubert et Mauss (Mauss, 1960) permettent de formaliser le processus métaphorique. Revoyons-les brièvement en rapport avec notre mythe.

La première opération recouvre les associations par contiguïté - «métonymie» - la réduction d'un tout à une de ses parties en vertu de l'appartenance même de cette dernière à une totalisation dont elle constitue un "échantillon » valable. (On peut évoquer ici comment toute communication humaine repose sur la réduction, opération sémiotique par excellence : si je décris mon meilleur ami, je le "réduis » à quelques traits que je considère fondamentaux selon le type de signifiance que je pratique. Quand je recueille une " histoire de vie», mon informateur réduit son existence à quelques heures. La théorie de la relativité réduit le cosmos en 
quelques pages, etc. $-c f$. la notion de « modèle réduit » de Lévi-Strauss [1962]. Et il en va de même en statistiques quand on réduit une population à un échantillon représentatif.)

Or, dans le cas du mythe qui nous occupe, on réduit le poisson à ses écailles, l'igname à ses découpures. Il va sans dire que cette réduction préalable à la deuxième opération s'avère déjà «précontrainte » (Lévi-Strauss 1962) par celle qui la suivra et qu'on anticipe - dont elle est " prégnante» pour utiliser le terme de René Thom.

La deuxième opération mise en relief par Hubert et Mauss porte sur l'association par similarité. Une fois deux objets quelconques réduits à une partie représentative du tout, on établit une relation de ressemblance entre les deux. On peut alors remonter de ces parties représentatives vers les touts qu'elles « résument », pour, "généralisant» à partir des " échantillons » prélevés, les déclarer équivalentes selon certains aspects. Nous avons là une "métaphore », notion exprimée en lau par le vocable kwaililifaila, qui signifie à la fois « imitation, écho, modèle »: kwai préfixe de réciprocité ; lili "déambuler»; fai avec ; la suffixe transformant un verbe en substantif.

Quant à la troisième opération dont traitent Hubert et Mauss, l'inversion, j'y reviendrai brièvement plus bas, avant de la reprendre éventuellement de façon plus développée, en rapport avec la formule canonique pour l'analyse des mythes (Lévi-Strauss 1958), dans une monographie dont ce texte ne fournit qu'un aperçu sommaire. Pour le moment, je reprends le sujet de la métaphore métamorphisante.

(2) Métaphore métamorphisante. Articulant la métaphore à la métonymie, on obtient une métaphore «efficace», « métamorphisante». En effet, repliant la deuxième opération sur la première, on retrouve l'association par contiguïté par delà l'association par similarité. La « métaphore » se ressource dans le tout de la réduction duquel on a tiré partie pour la concevoir. Elle s'articule au « réel » qu'elle investit. Elle devient alors métamorphisante, effectuant ce qu'elle signifie. Émanant du pragmatique, le signifiant - le sémantique - le frappe en retour de son sceau, comme, selon Victor Turner dans ses analyses du rituel, l'eau baptismale marque d'un sceau indélébile l'être qui devient chrétien.

Une fois la réduction effectuée d'un poisson à ses écailles - un poisson nécessairement de grande taille pour qu'il ait de grosses écailles comparables à des découpures d'ignames - et de l'igname à ses découpures, le mythe généralise pour associer l'un à l'autre et motiver la métamorphose. Mais d'abord peut-on mieux saisir le processus d'association par similarité, lequel, on le sait, précontraint le processus de réduction qui, quant à la « logique », le précède mais qui, quant à la pragmatique, le suit?

Comme noté en rapport avec I-1-35, l'igname ne se reproduit pas spontanément ; les variantes offrent donc une lecture à rebours de sa prolifération telle qu'on l'observe. On aura construit le mythe à partir d'une pratique courante, celle de détailler les tubercules en découpures, et on a perçu en elles une ressemblance aux écailles de l'alia. Cette association par similarité justifiera la réduction de ces deux touts à des parties permettant de les retotaliser l'un dans l'autre. Fort de cette analogie écailles coupures, un narrateur inventif construira le mythe en le fondant sur la constatation de similarité ( $c f$. la notion de «déduction empirique» Lévi-Strauss 1971). Ainsi, les découpures d'ignames construisent leur signifiance dans des écailles de poisson et, corrélativement, celles-ci construisent la leur dans celles-là. Le mythe trouvera sa genèse dans le développement, la narrativisation de la métaphore métamorphisante. Et à cette fin, il expliquera l'introduction de l'igname à Malaita selon un mode complexe.

En effet, comparons la version Kwara'ae (I-10), fort succincte, selon laquelle l'igname flotte tout simplement jusqu'à Malaita, à I-7 selon laquelle l'igname, animée, déjà poisson, coopère avec l'homme qui l'a pêchée et l'instruit sur la façon de la cultiver.

En principe, les Lau n'auraient-ils pas fort bien pu se contenter de la version I-10 ? Mais, dans leurs mythes d'origine, ils se montrent plus exigeants : de simples explications « réalistes » ne leur suffisent pas. Qu'ils traitent de l'origine de la noix de coco, du cochon, de la noix d'arec, etc., ils « dramatisent» au sens aristotélicien du terme. Nettement plus « symbolique » que I-10, I-7 dote l'igname d'automotion, et, comme la variante 'are'are, marque explicitement sa nature de poisson. Évidemment, I-1 va encore plus loin dans le processus de dramatisation. Son narrateur déploie la relation entre l'igname sous ses deux formes, la terrestre et la marine ; il développe la métamorphose d'un produit de la terre en produit de la mer et inversement. Et I-1, comme la plupart des autres variantes, montre la résistance de l'igname à s'implanter à Malaita, les difficultés qu'on éprouve à l'importer. J'y reviendrai. Pour le moment, contentons-nous de noter comment le mythe fonctionne en explicitation de la métaphore germinale, pleine de sens quand on la situe dans le contexte ethnographi- 
que esquissé dans la section 2 , surtout en ce qui a trait au rôle, sur les marchés, des femmes initiées.

Selon le commentateur de la variante du grand-prêtre de l'igname mentionné plus haut (section 3, (6)), la métamorphose implique une transsubstantiation effectuée par l'esprit. Son interprétation de l'enchaînement des métamorphoses se résume ainsi :

esprit $\Rightarrow$ igname $=>$ poisson $=>$ esprit $=>$ poisson $=>$ écailles $=>$ igname.

Serait-il valide de lire cette interprétation comme un dépouillement graduel des caractéristiques d'esprit de l'igname, de sorte que, lors de la dernière métamorphose - celle des écailles en découpures de tubercules - l'esprit ne conservait guère plus de charge extraordinaire que celle qui persiste dans les tabous qui entourent encore la manipulation du tubercule? Là-dessus, le commentateur indiqua cependant que la neutralisation du caractère surnaturel de l'igname ne connut son terme qu'une fois la première récolte rentrée. L'igname qu'on planta à l'origine conserva donc sa nature d'igname-esprit; mais celle qu'on obtint avec la première récolte devint une igname tout court, celle qu'on connaît aujourd'hui et dont les tabous qui l'entourent témoignent de son ancienne appartenance au monde des esprits.

Je mentionne ici que ce même commentateur opposa une fin de non-recevoir catégorique à ma suggestion que, dans les marchés, on transforme poissons en ignames et vice versa. Pour lui, cette transaction est d'un tout autre ordre que celle des métamorphoses de l'igname originelle, l'igname-esprit.

(3) Résistance de l'igname-poisson au renversement de la métamorphose. Il faut examiner aussi une autre réticence, celle de l'igname à recouvrer sa nature originelle après sa métamorphose en poisson. Préalablement, notons comment les détenteurs de l'igname essaient d'empêcher qu'on la leur prenne. Mais l'esprit du clan des voleurs s'avérera plus puissant que celui des premiers. Au contraire de son comportement dans I-7, l'igname-poisson des autres variantes manifestera la même résistance que celle de ses détenteurs originels à son implantation hors de son premier territoire.

Revenant paradoxalement à la charge pour se faire capturer tout en empêchant les pêcheurs de l'attraper, l'igname poisson se comporte comme si, perplexe quant à son " ontologie ", elle hésitait entre ses statuts marin et terrestre. Nous aurions là une dynamique de métaphore jouant une sorte $d$ '«indécision métamorphisante», taquinant l'esprit comme le poisson taquine les pêcheurs et les assoupit. Une métamorphose qui tarde délibérément à s'opérer, une métamorphose en gestation tergiversante pour ainsi dire : la sémiosis d'atermoiements d'un produit de la terre qui, une fois devenu marin, se montre réticent à retrouver sa condition première. Le mythe exprimerait par là une certaine tension entre produit de la terre et produit de la mer, une tension qu'on peut effectivement remarquer dans les marchés, surtout ceux du sud de la lagune, où les transactions, au lieu de se dérouler dans la bonhomie qui marque celles du centre de la lagune entre Lau et Baelelea ou Lau et Baegu, s'effectuent dans une certaine méfiance, voire avec une certaine agressivité entre Lau et Fataleka.

Mais une autre tension existe aussi entre Lau et ignames : ceux-là rejettent celles qui les incommodent et que je vais essayer de situer dans une architecture de rapports.

(4) Résistance de l'igname à la consommation. Le mythe (I-1 comme d'autres variantes) pose le problème $\mathrm{du}$ comportement face à certaines variétés d'ignames et rejette reetaba, pourtant la « mère » des ignames, de même que certaines de ses « filles ». En rapport avec les commentaires des lexicographes lau cités à propos de I-1-97 (supra, section 3 (13)), on peut recenser selon les variantes les causes de rejet pour en établir la hiérarchie d'après la gravité des inconvénients, rattachés surtout à reetaba.

(i) Igname bruyante, indiscrète, qui perturbe l'intimité : « Si nous raclons cette igname, il n'y a pas un homme, si loin soit-il, qui n'entende le bruit. Cette igname-là, la reetaba, nous l'avons renvoyée parce que le bruit était trop fort» (I-1-96).

D'une igname simplement bruyante, qui proclame à tout vent qu'on effectue des activités somme toute privées, on peut passer à une igname douée de parole qui, elle, dérange par ce comportement. Du bruit, "mécanique », d'une plante fonctionnant à la manière d'une caisse de résonance, on en vient à une propriété interne, la capacité d'émettre des sons spontanément, de parler, capacité troublante chez un cultigène.

(ii) Igname parlante : «Comme on ne veut pas obtempérer et qu'on trouve gênante une igname parlante, on renvoie la reetabailua chez elle à Alai » (I-6-12 (cf. aussi I-7-6 et I-8-7). Par là, on recoupe l'igname-esprit qu'on trouve dans une autre variante (I-5), condition qui lui permet de se métamorphoser en poisson ( $c f$. section 3 (13), ethno-étymologie (2) (b)) et d'endormir les pêcheurs (I-2-37). Cette igname-là, on ne la 
vend pas: «On ne vendit cependant que l'igname propre à la consommation, pas l'igname-esprit » (I-5-13).

(iii) Assurément, toute igname peut s'avérer «meurtrière " si on ne respecte pas les tabous qui entourent les contacts avec elle (section 3, (9)) mais seule reetaba représente une menace encore plus redoutable : celle d'aliment inversé puisque cannibale (les Lau se démarquent résolument des cannibales de la grande île). En outre, dans la variante du grand-prêtre de l'igname, ce tubercule provient du pays des goosile, monstres cannibales. Reetaba partagerait-elle leur appétit de chair humaine?

On aurait donc, selon les variantes, divers degrés de motivation du rejet de reetaba, allant d'un simple manque de discrétion au cannibalisme en passant par le fait insolite qu'elle puisse parler et aussi, fait encore plus inquiétant, donner la mort si on ne la traite pas avec de grands soins.

Somme toute, la sémiotique du mythe d'origine de l'igname à Malaita se ramène à une opération de déploiement selon les principes définis par Hubert et Mauss. De la réduction d'un ensemble à une de ses parties - réduction orientée et prédéfinie par l'association qu'on fera à un autre ensemble, également réduit à une de ses parties - , on s'achemine vers une métamorphose. Vers une métamorphose qui, par stratégie discursive, manifestera une certaine réticence à s'achever. Réticence à laquelle s'en ajoute une autre : celle de la consommation du tubercule. La prudence avec laquelle on finit par réussir à domestiquer une igname esprit révélerait qu'on fait face à un aliment symbolique tout autant que réel, aliment lourd de sens, aliment polysémique dont l'importance exige qu'on en dramatise judicieusement l'acquisition.

Je dois maintenant, en conclusion, contextualiser cette approche du mythe dans la sémiotique plus vaste de l'univers de représentations et comportements dont il émane et qu'il supporte.

\section{Conclusion}

Il faut ici reprendre en l'intégrant au reste la section 2 sur le sommaire de données ethnographiques relatives à l'igname. Je ne reviendrai pas systématiquement sur les données fournies là. Le lecteur n'a sans doute pas manqué de construire, à mesure qu'il avançait, sa propre analyse de la conception de l'igname chez les Lau. Ma conclusion ne fera donc vraisemblablement que confirmer sa démarche et son aboutissement.
Structure générative du mythe - mythogenèse.

«Déduction empirique » (Lévi-Strauss 1971) : selon la pragmatique,

(1) Découpures d'ignames $\Rightarrow$ plantation

Association par similarité visuelle :

(2) Ressemblance entre découpures et écailles de gros poissons

Métaphore, dérivée de (2) :

(3) écailles $=$ découpures

Métamorphose, par inférence de (3) :

(4) motivation de (3) : écailles $=>$ découpures

(5) inférence: pour que les écailles deviennent/donnent des découpures, il fallait que le poisson fût une igname, et il aura fallu que l'igname devînt préalablement poisson. Inversement, les découpures devraient se transformer en écailles, c'est-à-dire, les ignames en poissons, ce qui se passe sur les places de marchés.

Mythogenèse :

(6) d'où narrativisation, le mythe déployant la métaphore métamorphisante.

\section{Congruence :}

(7) association par contiguïté : l'igname représente métonymiquement tous les produits de la terre, l'alia tous les produits de la mer ;

(8) les besoins alimentaires et réciproques des gens de la terre et de ceux de la mer exigent une synergie qui, en pragmatique, a pris la forme de marchés ;

(9) d'où les métamorphoses de poissons en légumes dans le rituel d'initiation des jeunes femmes qui deviennent par là

(10) opératrices des transactions sur les marchés et

(11) conséquemment opératrices de synergie entre terre et mer.

\section{Jeu terre-mer}

Produit par excellence de la terre, l'igname comestible ; produit de la mer par excellence, un gros poisson comestible. L'accès obligé au premier dans la terre des esprits, se fait par voie marine ; la capture du second également. D'où prééminence de la mer sur la terre - du «thalassique » sur le chthonien. Tout se jouerait donc entre terre et mer. La mer, lien incontournable entre les îles océaniennes entre lesquelles, balises, elle se mesure, îles dont l'existence la cartographie, la définit comme espace et lui donne signifiance.

Revenons à de Coppet $(1976: 171 ; c f$. aussi Barraud, 1972). 
Ils sont nombreux les peuples mélanésiens qui pensent la récolte des ignames comme une pêche à la bonite, et celle-ci comme une chasse aux têtes ${ }^{4}$. Dans un tel système, la terre du jardin nourrit des poissons, que l'homme vient pêcher le jour de la récolte. La surface de la mer et celle $\mathrm{du}$ jardin sont deux espaces semblables où l'homme travaille à capturer des prises tirées des profondeurs : il s'active à ramener à la surface du sol et de la mer ou il vit, les éléments essentiels de sa subsistance, de ses fêtes, de ses dépenses, de ses destructions. Le jardin est un théâtre où l'homme parvient à franchir à son profit la ligne horizontale du sol qu'il foule de ses pieds nus. L'océan est l'autre jardin pour de semblables exploits.

Convertibilité de l'un et l'autre de ces deux " éléments», de ces deux " grappes de sens » entre lesquels les Lau dichotomisent le monde ; convertibilité par l'intermédiaire de leur métonymisations symétriques où l'une inverse l'autre, le tubercule devenant marin, le poisson devenant terrestre. Cela possible parce que l'igname survit dans la mer où elle flotte quand le poisson, lui, meurt sur terre; il n'y subsistera que par ses écailles, qui devront germer. A terre, donc, le poisson ne survit que sous forme d'igname. Et certaines de ses écailles fécondes donneront l'igname cannibale qui finira par se métamorphoser en pierre (I-9) - monument inerte et incomestible.

Mais restons-en à la synergie terre-mer, le produit de l'une devenant produit de l'autre et inversement. Une synergie qui s'exprime dans la réversibilité non seulement de la métaphore mais dans celle, d'une signifiance encore plus « profonde » parce que témoignant d'une synergie plus puissante, de la métamorphose. On a là l'acquisition d'un statut dimorphique, le produit de la terre des esprits se voyant englouti par la mer, s'y adapte pour devenir produit marin, avant de reprendre sa condition originelle tout cela par l'intermédiaire d'actions posées par des hommes, mais synergie que répercutent quotidiennement les femmes sur les marchés : synergie entre hommes et femmes sur quoi il faudra revenir plus bas (section suivante).

Je termine ces brèves considérations sur le jeu terre-mer en remettant en cause la priorité que Hviding (1992) assigne à la seconde. Et, dans le contexte des cadastres à tracer comme imposé par la loi sur la tenure foncière aux Salomons (héritage du colonialisme britannique), je dois mentionner les appropriations de terres par les Lau gagnés au système capitaliste, ces faafefe (littéralement ces «fais-moi chier ») qui, dérogeant à la tradition de communalité des biens et des usufruits, visent le profit personnel au détriment du maintien du délicat équilibre entre terre et mer qu'on avait toujours soigneusement maintenu ( $c f$. Schneider 1994).

\section{Jeu homme-femme}

\section{Appropriation masculine}

(1) Selon la leçon de I-1, ce produit par excellence de la terre, l'igname, existe dorénavant à Malaita à cause de l'initiative d'hommes qui vont la chercher dans un monde dangereux, et qui doivent s'en emparer contre le gré de ses détenteurs originels. Haut-fait d'hommes, donc. (2) Ce produit de la terre des esprits deviendra poisson en conséquence soit du fait que les esprits veulent en empêcher l'acquisition par des humains, soit parce que ses ravisseurs, le mettant à bord d'une même embarcation qu'elles selon la prévalence de la volonté de l'aîné sur celle du cadet, l'associent à des femmes par contiguïté. Les femmes deviennent ainsi la cause du chavirement, opérateurs involontaires de la condition de la métamorphose. (3) Affaire d'hommes, encore, que la récupération de l'igname devenue produit de la mer - la pêche relevant exclusivement d'eux. Hommes qui devront déjouer et venir à bout de la résistance de l'igname à se laisser capturer et à recouvrer sa forme originale, en jetant les pêcheurs dans le sommeil. (4) Affaire d'hommes, toujours, que la plantation et la culture de l'igname. (5) Enfin - on peut le conjecturer - l'origine du rituel d'initiation à la nubilité des jeunes femmes relèverait des hommes, comme d'ailleurs, peut-être, l'origine de tous les autres rituels.

\section{Appropriation féminine}

Mais alors les hommes, ne pouvant échapper à une « ontologie » qui les envahit en autant qu'ils et parce qu'ils la définissent, se remettent euxmêmes dans les mains des femmes, au propre comme au figuré. D'abord, ils n'acquièrent leur plein statut qu'après avoir fait couler « du haut » d'une victime, et en risquant leur vie, un sang qui, chez les femmes, s'échappe spontanément « du bas ». Et ce sang, ils le font couler grâce au clitoris d'ébène qui, les transformant en femmes figurées, leur sert d'arme. A ce propos, on a là une autre réduction (métonymie); de la femme à son clitoris, lequel, façonné en massue, l'homme s'approprie symboliquement et par contiguité. Clitoris qui, porté par l'homme sur son épaule,

4. Un clan lau utilise une sculpture de bonite dans le corps de laquelle on ménage un trou pour y entreposer le crâne du dernier grand-prêtre décédé. 
lui surplombe la tête. L'homme ne peut s'approprier lui-même que par sa soumission à la partie la plus dangereuse de la femme.

Ensuite, ils remettent à leurs femmes (sœurs, filles nubiles, mères...) le produit de leur pêche pour qu'elles en disposent à leur gré sur les marchés. L'homme a beau demander à sa femme, par exemple, de vendre ses poissons pour des dents de dauphin en vue d'une contribution à une compensation matrimoniale, un rituel, une fête, elle n'en fera qu'à sa tête et, souvent, échangera plutôt les poissons de son ou ses hommes d'abord pour des légumes - cela s'impose pour assurer l'alimentation de base - , et ensuite pour du tabac, des fruits, des noix d'arec, selon son bon plaisir à elle.

Transformant des produits de la mer en produits de la terre, utilisant les marchés comme complément aux petits jardins des Lau - voire comme un super-jardin -, la femme transforme pour ainsi dire son mari de pêcheur au premier degré en agriculteur au second degré : elle métamorphose un marin en terrien. Gestionnaire de la vie quotidienne, elle ne lui laisse plus que le champ du rituel pour exprimer une condition fragile, constamment menacée par celles qu'il redoute au point de confiner en réclusion lors de ces temps forts et si dangereux des menstrues et des accouchements, celles qui, lui ayant donné naissance et perpétuant son clan, peuvent encore plus facilement lui donner la mort et causer l'extinction de sa lignée.

Mais, de toute façon, de nos jours, la loi d'enregistrement des sols et la christianisation réussissent à neutraliser les femmes, à les rendre inoffensives. L'enseignement des missionnaires chrétiens ayant rendu plusieurs payens agnostiques d'une part (Maranda 1979, 2001), et, de l'autre, promouvant la suprématie des hommes sur les femmes, ces dynamiques d'importation ont rompu tout aussi bien le délicat équilibre entre les sexes que celui entre gens de la terre et gens de la mer.

\section{RÉFÉRENCES}

BARrAU, Jacques, 1956. L'Agriculture vivrière autochtone de la Nouvelle-Calédonie. Nouméa : Commission du Pacifique Sud.

—, 1965. L'humide et le sec: An Essay on ethnobiological adaptation to contrastive environments in the Indo-Pacific area. Journal of the Polynesian Society 74 (3) : 329-346.

—, 1967. Les hommes, les plantes et la mer en Océanie tropicale. Cahiers du Pacifique ${ }^{\circ} 10$ : 59-78.
BARraud, C., 1972. De la Chasse aux têtes à la pêche à la bonite. L'Homme XII : 67-104.

Bonnemaison, Joël 1991. Magic Gardens in Tanna. Pacific Studies 14 (4) : 71-89.

_, 1996. Les Fondements géographiques d'une identité. L'Archipel du Vanuatu. Essai de géographie culturelle, Livre 1 Gens de pirogue et gens de la terre. Paris : ORSTOM.

_, 1997. Les Fondements géographiques d'une identité. L'Archipel du Vanuatu. Essai de géographie culturelle, Livre I1 - Les Gens des lieux. Histoire et géosymboles d'une société enracinée : Tanna. Paris : ORSTOM.

Coppet, D. de, 1976. Jardins de vie, jardins de mort en Mélanésie. Traverses 5/6 : 166-177.

Godelier, M., 1996. L’Énigme du don. Paris: Fayard.

Granada Television, 1987. The Lau of Malaita. Film réalisé par Leslie Woodhead et Pierre Maranda, Série « Disappearing World».

Hymes, D., 1971. The «Wife » Who « Goes Out» Like a Man: Reinterpretation of a Clackamas Chinook Myth, in Maranda, P, and E. Köngäs Maranda (eds), Structural Analysis of Oral Tradition. Philadelphia: University of Pennsylvania Press, pp. 49-80.

Hviding, E. 1992. Guardians of Morovo Lagoon. The Sea as Cultural and Relational Focus in New Georgia, Solomon Islands. Bergen: University of Bregen, Ph. D. Thesis.

Ivens, W.G, 1927. Melanesians of the S. E. Solomon Islands. London: Kegan Paul.

LÉvi-Strauss, C., 1958. Anthropologie structurale. Paris: Plon.

_, 1962. La Pensée sauvage. Paris: Plon.

_, 1966. Le Cru et le cuit. Paris: Plon.

—, 1971. The Deduction of the Crane, in Maranda, P, and E. Köngäs Maranda (eds), Structural Analysis of Oral Tradition. Philadelphia: University of Pennsylvania Press, pp. 3-21.

Malinowski, B., 1922. Argonauts of the Western Pacific. London: Routledge and Kegan Paul.

-, 1935. Coral Gardens and their Magic. London: George Allen \& Unwin.

MarandA, P., 1970. Of Bears and Spouses: Transformational Analysis of a Myth, in E. Kongas Maranda and P. Maranda, Structural Models in Folklore and Transformational Essays, 2nd ed. Paris-The Hague: Mouton, ch. 3, pp. 95-115.

—, 1979. Situer l'anthropologie, in L.-J. Dorais, Perspectives anthropologiques. Un Collectif d'anthropologues québécois. Montréal: Éditions du Renouveau Pédagogique, pp. 9-22, 399-436.

- 1980. The Dialectics of Metaphor: An Essay in Anthropological Hermeneutics, in Suleiman, S. and I. Crosman, The Reader in the Text. Princeton: Princeton University Press, pp. 183-204. 
_, 1982a. Anthropological Analytics, in I. Rossi, The Logic of Culture. Advances in Structural Theory and Methods. South Hatley, Mass.: Bergin Publishers, pp. 23-41.

—, 1982b. Semiotik und Anthropologie. Zeitschrift für Semiotik 3, pp. 227-249.

—, 1985. Un Ici ailleurs. Pourquoi faire du terrain ? in S. Genest, La Passion de l'échange: Terrains d'anthropologues du Québec. Chicoutimi, Qc: Gaétan Morin, pp. 101-108 [reproduit in Québec Science, 24, 4 (1985), pp. 22-27].

—, 1997a. A Semiotic Encyclopedia of the Lau People (Malaita, Solomon Islands). Computer Diskette. Québec: Laboratoire de recherche sémiographique en anthropologie.

_, 1997b Metaforas metamorficas: operadores que aplicam cultura ao comportamento, in Monica Rector, ed., Comunicaçao na era pos-moderna. Petropolis: Editora Vozes, pp. 116-127.

_, 1997c. Superheroines in Lau Myth and Society: Womankind as the Architectonic Principle of Ontology. Paper presented in the ASAO Working Session "Superheroines: Mythology and Gender in the Pacific", San Diego, USA..

-, 2001. Mapping Historical Transformation Through the Canonical Formula: The Pagan vs. Christian Ontological Status of Women in Malaita, Solomon Islands, in P. Maranda (ed.) The Double Twist: From Ethnography to Morphodynamics. Toronto: The University of Toronto Press. pp. 97121.
—, sous presse. Echo chambers and rhetoric. Sketch of a model of resonance theory, in Y. Strecker and Christian Meywr (eds), Rhetoric Culture : General Theory. Mainz: Institute for Ethnology and African Studies.

Maranda, P. et E. Köngäs Maranda, 1970. Le Crâne et l'utérus: Deux Théorèmes nordmalaitains, in Pouillon, J. et P. Maranda, Échanges et communications, 2 vol. Paris: Mouton, vol 2. pp. 829-861.

Marshall, T.C., 1964. Fishes of the Great Barrier Reef and Coastal Waters of Queensland. Sydney, Australia: Angus and Robertson.

Mauss, M., 1960. Avec Hubert, H., Esquisse d'une théorie générale de la magie, Sociologie et anthropologie. Paris: Presses Universitaires de France, pp. 3-144.

Panoff, F., 1969. Some facets of Maenge Horticulture. Oceania 40.

PANOFF, M., 1970a. Father Arithmetic: Numeration and Counting in New Britain. Ethnology 9, 4, pp. 358-365.

—, 1970b. Food and Faeces: A Melanesian Rite. Man 5 , pp. 237-251.

SCHNEIDER, G., 1994. Land Disputes Reflecting Changing Concepts of Traditional Culture. Cambridge: Department of Social Anthropology, Dactylogramme. 